Discussion Paper No. 629

\title{
PAIRWISE STRATEGY-PROOFNESS
}

\section{AND SELF-ENFORCING MANIPULATION*}

\author{
Shigehiro Serizawa
}

February 2005

The Institute of Social and Economic Research Osaka University

6-1 Mihogaoka, Ibaraki, Osaka 567-0047, Japan

*Forthcoming in Social Choice and Welfare 


\title{
Pairwise Strategy-Proofness and Self-Enforcing Manipulation
}

\author{
By Shigehiro Serizawa ${ }^{1}$
}

\author{
Institute of Social and Economic Research, Osaka University \\ 6-1, Mihogaoka, Ibaraki, Osaka 567-0047, Japan \\ E-mail: serizawa@iser.osaka-u.ac.jp \\ Phone: (+81) 6-6879-8558 \Fax: (+81) 6-6878-2766
}

\begin{abstract}
Strategy-proofness" is one of the axioms that are most frequently used in the recent literature on social choice theory. It requires that by misrepresenting his preferences, no agent can manipulate the outcome of the social choice rule in his favor. The stronger requirement of "group strategy-proofness" is also often employed to obtain clear characterization results of social choice rules. Group strategy-proofness requires that no group of agents can manipulate the outcome in their favors. In this paper, we advocate "effective pairwise strategy-proofness." It is the requirement that the social choice rule should be immune to unilateral manipulation and "self-enforcing" pairwise manipulation in the sense that no agent of a pair has the incentive to betray his partner. We apply the axiom of effective pairwise strategy-proofness to three types of economies: public good economy, pure exchange economy, and allotment economy. Although effective pairwise strategy-proofness is seemingly a much weaker axiom than group strategy-proofness, effective pairwise strategy-proofness characterizes social choice rules that are analyzed by using different axioms in the literature.
\end{abstract}

\footnotetext{
${ }^{1}$ I thank Professors S. Barberà, T. Saijo, J. Schummer and anonymous referee for their useful comments. I also thank W. Kureishi for his detailed comments and R. Serizawa her careful reading.
} 


\section{Introduction}

When a collection of individuals has to choose an allocation, the procedure they use for making their choice should take into account their preferences. Procedures are formally represented as functions from the class of possible preference profiles into the feasible set, and they are called social choice functions, or social choice rules. In this paper, we call them rules for short. "Strategy-proofness" is one of the axioms that are most frequently used in recent literature on social choice theory; it says that by misrepresenting his preferences, no agent can manipulate the outcome of the rule in his favor ${ }^{2}$. "Group strategy-proofness" is also often employed to obtain clear characterization results of rules. This condition is a much stronger requirement than strategy-proofness in that a rule must be immune to strategic misrepresentation of preferences by all kinds of coalitions of agents. Since cooperation in such manipulation is difficult in many situations, group strategy-proofness is considered to be unnecessarily demanding. Thus, one might suspect that desirable properties of rules are unnecessarily sacrificed for the requirement of group strategy-proofness.

In this paper, we advocate "effective pairwise strategy-proofness". This condition weakens group strategy-proofness by restricting manipulations to which rules must be immune as follows. Cooperation in strategic misrepresentation is especially difficult for large coalitions. Thus, effective pairwise strategy-proofness does not require that a rule should be immune to manipulations by more than two agents. Note that in pairwise manipulation, there is an incentive for an agent to betray his partner to increase his utility further. If there is a chance of such betrayal, a pairwise manipulation is not "selfenforcing" ${ }^{3}$, and the society may not have to worry about pairwise manipulations that are not self-enforcing. Therefore, effective pairwise strategy-proofness neglects non-selfenforcing pairwise manipulations. That is, effective pairwise strategy-proofness is a requirement that a rule should be immune only to unilateral manipulation and self-enforcing pairwise manipulation ${ }^{4}$. Accordingly, although effective pairwise strategy-proofness is stronger than strategy-proofness, it is a reasonable requirement. We apply effective pairwise strategy-proofness to various kinds of economies, such as public good economies, pure exchange economies, and allotment economies. As a result, we obtain new characterizations of classes of rules those are analyzed in literature.

In their influential articles, Gibbard (1977) and Satterthwaite (1975) characterize dictatorships ${ }^{5}$ to be the only strategy-proof rules on the universal domain ${ }^{6}$ where there are at least three alternatives in the range. Since then, many authors employ strategyproofness to characterize various classes of rules in different kinds of models. For example, Moulin (1980) and his successors identify the class of strategy-proof rules for economies where the feasible set consists of only public alternatives and agents have single-peaked ${ }^{7}$

\footnotetext{
${ }^{2}$ In game theoretic terminology, a rule is strategy-proof if it is a weakly dominant strategy for each agent to represent his true.

${ }^{3}$ This notion of self-enforcing is similar to self-enforcingness of Peleg and Sudhölter (1999).

${ }^{4}$ In this paper, we do not consider the private transfer between agents. Schummer (2000) independently analyzes the joint manipulation of two agents when the transfer between agents is possible in economies where preferences are quasi-linear.

${ }^{5} \mathrm{~A}$ rule is a dictatorship if there is some agent such that the outcome of the rule is always his most preferred element in the feasible set.

${ }^{6}$ The universal domain is the class of all linear preferences on the feasible set.

${ }^{7} \mathrm{~A}$ preferences is single-peaked if there is some point, called "peak", and alternatives closer to the peak are more preferred.
} 
preferences. However, when there is at least one private good, strategy-proofness alone is not strong enough to obtain meaningful classes of rules. Thus, together with some additional properties, conditions stronger than strategy-proofness are often required in characterizing rules.

Moulin (1994) provides a remarkable example of public good economies. He applies group strategy-proofness and symmetry ${ }^{8}$ to economies with one public good and one private good. As a result, he obtains "equal cost share rules," that is, rules where agents equally share the cost of the public good, regardless of whatever preferences they may have.

Barberà and Jackson (1995) also provide a notable example in pure exchange economies. They employ "nonbossiness" introduced by Satterthwaite and Sonnenschein (1981) together with strategy-proofness. The condition of nonbossiness says that by changing his announced preferences, no agent can change the other agents' consumption bundles without changing his own consumption bundle. When combined with strategy-proofness, the requirement of nonbossiness implies a weak variant of group strategy-proofness called "weak group strategy-proofness" on the class of classical preferences. Using this fact, Barberà and Jackson succeed in identifying the class of strategy-proof, nonbossy, and anonymous ${ }^{9}$ rules for pure exchange economies.

In this paper, first, we apply effective pairwise strategy-proofness to public good economies where there are one private good and one pure public good. We assume that the domain of preferences is classical or it is quasi-linear. We establish that if a rule is effectively pairwise strategy-proof, then it is a "preference independent cost share rule"; each agent shares the cost of the public goods according to his own cost share function that depends only on the quantity of the public goods produced. When such cost share functions are considered to reflect some social norm such as ability to pay, this result supports the ability to pay doctrine against benefit doctrine. We also establish that effective pairwise strategy-proofness is equivalent to group strategy-proofness on the class of classical preferences in public goods economies.

Second, we apply effective pairwise strategy-proofness to pure exchange economies where preferences are classical, homothetic and smooth. We establish that there is no effectively pairwise strategy-proof, Pareto-efficient, and nondictatorial rule. As corollaries of this result on the class of classical, homothetic and smooth preferences, the same conclusions hold on any superdomain of the class of those preferences. It is also follows from this result that effective pairwise strategy-proofness together with Pareto-efficiency imply group strategy-proofness on the class of classical, homothetic and smooth preferences in pure exchange economies

Third, we apply effective pairwise strategy-proofness to allotment economies where there is a fixed amount of one private good that is not freely disposable, and preferences are single-peaked. We establish that a rule is effectively pairwise strategy-proof and symmetric, and respects unanimity ${ }^{10}$ if and only if it is the "uniform rule (Benassy, 1982)". We also establish that effective pairwise strategy-proofness together with respect

\footnotetext{
${ }^{8} \mathrm{~A}$ rule is symmetric if whenever two agents have the same preference, they receive the same consumption bundle.

${ }^{9} \mathrm{~A}$ rule is anonymous if whenever the preferences of two agents are switched, their consumption bundles are also switched.

${ }^{10}$ A rule in allotment economies respects unanimity if whenever the sum of agents' preferred levels is equal to the total endowment, agents receive their preferred levels.
} 
for unanimity imply group strategy-proofness on the class of single-peaked preferences in allotment economies.

We organize the paper as follows: In Section 2, we describe the general model and introduce basic notions. We state the results of the three types of economies, public good economies, pure exchange economies, and allotment economies in Sections 3, 4, and 5 respectively. We gather the proofs of all results in Section 6 .

\section{The General Model and The Basic Notions}

There are $n \geq 2$ agents. We denote the set of agents by $N=\{1,2, \ldots, n\}$. A coalition is a subset $N^{\prime}$ of $N$. Given a coalition $N^{\prime} \subseteq N$ and an agent $i \in N$, we denote the coalition $N \backslash N^{\prime}$ by $-N^{\prime}$ and the coalition $N \backslash\{i\}$ by $-i$. There are $m_{X} \geq 1$ private goods and $m_{Y}$ (pure) public goods, where $m_{Y}$ is 0 or 1 . Let $m=m_{X}+m_{Y}$. We denote the set of goods by $M=\{1,2, \ldots, m\}$, and that of private goods by $M_{X}=\left\{1,2, \ldots, m_{X}\right\}$. Each agent $i$ is faced with his consumption set $Z^{i}=X^{i} \times Y=\mathbb{R}_{+}^{m}$, where $X^{i}=\mathbb{R}_{+}^{m_{X}}$ is the set of agent $i$ 's private good consumption bundles and $Y=\mathbb{R}_{+}$is the set of the public good levels. His consumption bundle $z^{i}=\left(z_{1}^{i}, \ldots, z_{m}^{i}\right)=\left(x^{i}, y\right)$ is an element of $Z^{i}$. The feasible set $Z$ is a subset of $X^{1} \times \cdots \times X^{n} \times Y$, and is specified for various types of economies in the following sections. An allocation $z=\left(x^{1}, \ldots, x^{n}, y\right)$ is an element of $Z$. Let $e=\left(e^{1}, \ldots, e^{n}, 0, \ldots, 0\right) \in Z$ be the endowment point, where $e^{i} \in \mathbb{R}_{+}^{m_{X}}$ is agent $i$ 's endowment of the private goods. Let $U^{0}$ be the class of preferences on $\mathbb{R}_{+}^{m}$ which are represented by continuous utility functions. We shall often abuse language and identify preferences with the continuous utility functions that represent them.

Definition. Let $U^{0 \prime} \subset U^{0}$, and $U=\left(U^{0 \prime}\right)^{n}$. A rule on $U$ is a function $f$ from $U$ to $Z$. The set $U$ is called the domain of $f$.

Definition. A preference $u^{i} \in U^{0}$ is classical if its utility function is continuous on $\mathbb{R}_{+}^{m}$, and it is strictly quasi-concave and strictly monotonic on the interior of $\mathbb{R}_{+}^{m}$.

We denote the class of classical preferences by $U^{C}$, and call it the classical domain.

Other types of domains are introduced in subsequent sections. Although agent $i$ 's utility function $u^{i}\left(x^{1}, \ldots, x^{n}, y\right)$ actually depends only on $z^{i}=\left(x^{i}, y\right)$, we treat $u^{i}$ as a function on $\mathbb{R}_{+}^{n \cdot m_{X}+m_{Y}}$ when it simplifies notation without creating confusion. We write $U=U^{1} \times \cdots \times U^{n}$, where $U^{i}$ is the set of agent $i$ 's utility functions. A preference profile is an element of $U$. Given $N^{\prime} \subseteq N$, let $U^{N^{\prime}}=\prod_{j \in N^{\prime}} U^{j}$. We denote generic elements of $U, U^{N^{\prime}}$ and $U^{-i}$ by $u, u^{N^{\prime}}$ and $u^{-i}$ respectively. If $u=\left(u^{1}, \ldots, u^{n}\right) \in U, N^{\prime} \subseteq N$, and $i \in N$ are given in advance, $u^{N^{\prime}}$ denotes $\left(u^{j}\right)_{j \in N^{\prime}}$ and $u^{-i}$ denotes $\left(u^{j}\right)_{j \in N \backslash\{i\}}$. Given $i \in N$ and $u \in U$, let the best element set $B^{i}(u)=\left\{z \in Z \mid \forall z^{\prime} \in Z, u^{i}(z) \geq u^{i}\left(z^{\prime}\right)\right\}$. Given a rule $f: U \rightarrow Z$ and a preference profile $u \in U$, we write $f(u)=\left(f_{1}(u), \ldots, f_{n}(u)\right.$, $\left.f_{y}(u)\right), f^{i}(u)=\left(f_{i}(u), f_{y}(u)\right)$, and $f^{-i}(u)=\left(\left(f_{j}(u)\right)_{j \neq i}, f_{y}(u)\right)$.

Definition. Given a $u \in U$, an allocation $z \in Z$ is Pareto-efficient for $u$ if for any $z^{\prime} \in Z$,

$\left[\exists i \in N\right.$ such that $\left.u^{i}\left(z^{\prime}\right)>u^{i}(z)\right] \Rightarrow\left[\exists j \in N\right.$ such that $\left.u^{j}(z)>u^{j}\left(z^{\prime}\right)\right]$.

A rule $f$ is Pareto-efficient if for any $u \in U, f(u)$ is Pareto efficient for $u$.

Next we consider distributional requirements of rules. A rule is "individually rational" if it never assigns an allocation which makes some agent worse off than he would be by consuming his endowment. This condition requires that all the agents should share in the 
fruit of cooperation. A rule is "symmetric" if two agents receive the same consumption bundle whenever they have the same preference; and it is "anonymous" if when the preferences of two agents are switched, their assigned consumption bundles are switched as well. These two conditions require a rule not to treat agents unfairly.

Definition. A rule $f$ is individually rational if for any $u \in U$ and any $i \in N, u^{i}(f(u)) \geq$ $u^{i}(e)$.

It is symmetric if for any $u \in U$, any $i \in N$, and any $j \in N$, $u^{i}=u^{j} \Rightarrow f_{i}(u)=f_{j}(u)$.

It is anonymous if for any $u \in U$, any $i \in N$, any $j \in N$, any $\hat{u}^{i} \in U^{i}$, and any $\hat{u}^{j} \in U^{j}$, $\left[\hat{u}^{i}=u^{j} \& \hat{u}^{j}=u^{i}\right] \Rightarrow\left[f^{i}\left(\hat{u}^{i}, \hat{u}^{j}, u^{-\{i, j\}}\right)=f^{j}(u) \& f^{j}\left(\hat{u}^{i}, \hat{u}^{j}, u^{-\{i, j\}}\right)=f^{i}(u)\right]$.

Definition. A rule $f$ is dictatorial if there is an agent $i \in N$ such that for any $u \in U$, $f^{i}(u) \in B^{i}(u)$; otherwise $f$ is nondictatorial.

The condition of "individual strategy-proofness" requires that a rule should be immune to the strategic behavior of any single agent; no agent can increase his utility by unilateral manipulation. Hereafter we call the condition of individual strategy-proofness simply "strategy-proofness" for short. "Group strategy-proofness" is a stronger condition; it requires that by coalitional manipulation no coalition can increase the utility of any member in the coalition without decreasing the utility of some other member in it. "Pairwise strategy-proofness" is an intermediate condition; it is stronger than individual strategy-proofness but weaker than group strategy-proofness. Pairwise strategy-proofness requires that no pair of agents can increase the utility of any agent of the pair without decreasing the utility of the other of the pair. A pairwise manipulation is "self-enforcing" if the manipulation does not decrease the utility of either of the pair, increases the utility of at least one of the pair, and neither of the pair has the incentive to betray the partner. "Effective pairwise strategy-proofness" requires that no pair of agents should have a self-enforcing manipulation in addition to strategy-proofness. Thus, effective pairwise strategy-proofness is stronger than strategy-proofness, but weaker than pairwise strategyproofness.

Definition. A rule $f$ is (individually) strategy-proof if for any $u \in U$, any $i \in N$, and any $\hat{u}^{i} \in U^{i}, u^{i}(f(u)) \geq u^{i}\left(f\left(\hat{u}^{i}, u^{-i}\right)\right)$.

It is weak group strategy-proof if for any $u \in U$, any $N^{\prime} \subseteq N$, and any $\hat{u}^{N^{\prime}} \in U^{N^{\prime}}$,

$\exists i \in N^{\prime}$ s.t. $u^{i}(f(u)) \geq u^{i}\left(f\left(\hat{u}^{N^{\prime}}, u^{-N^{\prime}}\right)\right)$.

It is group strategy-proof if for any $u \in U$, any $N^{\prime} \subseteq N$, and any $\hat{u}^{N^{\prime}} \in U^{N^{\prime}}$, $\left[\exists i \in N^{\prime}\right.$ s.t. $\left.u^{i}\left(f\left(\hat{u}^{N^{\prime}}, u^{-N^{\prime}}\right)\right)>u^{i}(f(u))\right] \Rightarrow\left[\exists j \in N^{\prime}\right.$ s.t. $\left.u^{j}\left(f\left(\hat{u}^{N^{\prime}}, u^{-N^{\prime}}\right)\right)<u^{j}(f(u))\right]$.

Definition. A rule $f$ is pairwise strategy-proof if for any $u \in U$, any $N^{\prime}=\{i, j\} \subseteq N$, and any $\hat{u}^{N^{\prime}} \in U^{N^{\prime}}$,

$\left[u^{i}\left(f\left(\hat{u}^{N^{\prime}}, u^{-N^{\prime}}\right)\right)>u^{i}(f(u))\right] \Rightarrow\left[u^{j}\left(f\left(\hat{u}^{N^{\prime}}, u^{-N^{\prime}}\right)\right)<u^{j}(f(u))\right]$.

Definition. Given a rule $f$, a preference profile $u \in U$, and a pair of agents $N^{\prime}=\{i, j\}$, a preference profile of the pair $\hat{u}^{N^{\prime}} \in U^{N^{\prime}}$ is a self-enforcing manipulation if

(i) $u^{i}\left(f\left(\hat{u}^{N^{\prime}}, u^{-N^{\prime}}\right)\right) \geq u^{i}(f(u))$ and $u^{j}\left(f\left(\hat{u}^{N^{\prime}}, u^{-N^{\prime}}\right)\right) \geq u^{j}(f(u))$,

(ii) $u^{i}\left(f\left(\hat{u}^{N^{\prime}}, u^{-N^{\prime}}\right)\right)>u^{i}(f(u))$ or $u^{j}\left(f\left(\hat{u}^{N^{\prime}}, u^{-N^{\prime}}\right)\right)>u^{j}(f(u))$,

(iii) for any $\widetilde{u}^{i} \in U^{i}, u^{i}\left(f\left(\hat{u}^{N^{\prime}}, u^{-N^{\prime}}\right)\right) \geq u^{i}\left(f\left(\widetilde{u}^{i}, \hat{u}^{j}, u^{-N^{\prime}}\right)\right)$, and

(iv) for any $\widetilde{u}^{j} \in U^{j}, u^{j}\left(f\left(\hat{u}^{N^{\prime}}, u^{-N^{\prime}}\right)\right) \geq u^{i}\left(f\left(\widetilde{u}^{j}, \hat{u}^{i}, u^{-N^{\prime}}\right)\right)$. 
A rule is effectively pairwise strategy-proof if (i) it is strategy-proof, and (ii) no pair of agents has a self-enforcing manipulation.

Generally we say that an agent is "bossy" if he intervenes in what he is not concerned with. Since agent $i$ 's preference depends only on $z^{i}$, agent $i$ is not concerned with $z^{-i}$. Thus here, "nonbossiness" implies that by changing his announced preferences, no agent can change the other agents' consumption bundles $z^{-i}$ without changing his consumption bundle $z^{i}$.

Definition. A rule $f$ is nonbossy if for any $u \in U$, any $i \in N$, and any $\hat{u}^{i} \in U^{i}$, $f^{-i}(u) \neq f^{-i}\left(\hat{u}^{i}, u^{-i}\right) \Rightarrow f^{i}(u) \neq f^{i}\left(\hat{u}^{i}, u^{-i}\right)$.

Nonbossiness is introduced in Satterthwaite and Sonnenschein (1981). They discuss its economic motivation. This condition also plays a technically important role in our paper. Propositions 1, 3, and 5 in subsequent sections state that nonbossiness is closely related to effective pairwise strategy-proofness. In Section 4, however, we cite an example which emphasizes that effective pairwise strategy-proofness is mathematically independent of nonbossiness in pure exchange economies. That example also demonstrates that the two conditions are also mathematically independent in allotment economies.

\section{Public Good Economies}

In this section, we consider economies where there are one private good and one public good. Thus, $m_{X}=m_{Y}=1$ in this section. The public good is produced using the private good as an input which is collected from agents. The cost function $C$ of the public good is an increasing function from $\mathbb{R}_{+}$to $\mathbb{R}_{+}$. The feasible set $Z$ in this section is the set $\left\{z=\left(x^{1}, \ldots, x^{n}, y\right) \in X^{1} \times \cdots \times X^{n} \times Y \mid C(y) \leq \sum_{i \in N}\left(e^{i}-x^{i}\right)\right\}$. Given a rule $f$ on a domain $U$, we denote the production range of $f$ by $Y_{f}$, that is, $Y_{f}=\{y \in Y \mid \exists u \in U$ such that $\left.f_{y}(u)=y\right\}$.

Definition. A preference $u^{i} \in U^{C}$ is quasi-linear if there is a value function $v^{i}: \mathbb{R}_{+} \rightarrow \mathbb{R}_{+}$ such that

$$
\begin{aligned}
& \forall z^{i}=\left(x^{i}, y\right) \in Z^{i}, \forall \hat{z}^{i}=\left(\hat{x}^{i}, \hat{y}\right) \in Z^{i}, \\
& u^{i}\left(z^{i}\right) \geq u^{i}\left(\hat{z}^{i}\right) \Longleftrightarrow v^{i}(y)+x^{i} \geq v^{i}(\hat{y})+\hat{x}^{i} .
\end{aligned}
$$

We denote the class of quasi-linear by $U^{Q}$, and call it the quasi-linear domain.

How agents should share the cost of the public good has been discussed by even classical economists or earlier. One of influential doctrines was "ability to pay doctrine"; it insists that agents should share the cost of the public good according to their ability to pay, not the benefit they receive from the public good. However, since Samuelson (1954) analyzed the Lindahl mechanism, the modern economic theory pay most attention to rules based on "benefit doctrine"; it insists that the cost share of agents should depend on the benefit they receive. ${ }^{11}$ As we mentioned in the Introduction, Moulin (1994) employs the condition of group strategy-proofness together with symmetry to obtain "equal cost share rules," that is, the rules where agents equally share the cost of the public good, regardless of whatever preferences they may have. He establishes that if a rule is group strategy-proof and symmetric, then it is an equal cost share rule. Equal cost share rules belong to the class of "preference independent cost share rules." The idea of preference independent

\footnotetext{
${ }^{11}$ See Musgrave (1985) for the details of the literature.
} 
cost share rule is similar to ability to pay doctrine. Under a preference independent cost share rule, each agent has his own "norm cost share function", which is mathematically formulated as a function from $Y$ to $\mathbb{R}_{+}$, and which assigns his cost share for any level of the public good produced. Norm cost share functions are interpreted as reflecting the ability to pay of agents or a certain kind of social norm. Preference independent cost share rules are rules such that agents share the cost of the public good according to their norm cost share functions.

Definition. A rule $f$ is a preference independent cost share rule if there is a list of norm cost share functions $t_{i}: Y \rightarrow \mathbb{R}_{+}, i \in N$, such that for any $u \in U, C\left(f_{y}(u)\right) \leq$ $\sum_{i \in N} t_{i}\left(f_{y}(u)\right)$; and for any $u \in U$ and any $i \in N, f_{i}(u)=e^{i}-t_{i}\left(f_{y}(u)\right)$.

Under preference independent cost share rules, agents' preferences have influence on the levels of the public good produced, and thus the preferences have indirect influence on the cost shares the agents need to pay. However, the change of the preferences has no impact on the cost shares unless the level of the public good produced also changes. Proportional cost share rules illustrated in Example 1 below are an example of preference independent cost share rules.

Example 1 (Proportional Cost Share Rule). Let the number of agents $n$ be odd, and let $\alpha=\left(\alpha^{1}, \ldots, \alpha^{n}\right) \in \mathbb{R}_{+}^{n}$ be such that $\sum_{i \in N} \alpha^{i}=1$. Consider a rule $f$ such that for any $u \in U$ and any $i \in N, f_{i}(u)=e^{i}-\alpha^{i} \cdot C\left(\left(f_{y}(u)\right)\right.$. Then $f$ is a preference independent cost share rule. Assume further that $C$ is convex, and that the production range, $Y_{f}$, is convex. Then agents' classical and quasi-linear preferences induce single-peaked preferences on $Y_{f}$, and the peaks are agents' demands for the public good. If the public good production level is determined by "the median voter scheme", that is, if the public good is produced as much as the median of the demands, then $f$ is group strategy-proof.

Since equal cost share rules are considered to be based on ability to pay doctrine in the case that all the agents have the equal ability to pay, Moulin's (1994) result supports ability to pay principle from the view point of strategic behavior. Serizawa (1996, 1999), Ohseto (1997), Deb and Ohseto (1999) etc. succeed in strengthening his result by using strategy-proofness. However, some additional conditions such as symmetry, budgetbalancing, individual rationality, nonbossiness, etc. are still required to obtain preference independent cost share rules. In this section, we establish that effective pairwise strategyproofness alone is enough to obtain preference independent cost share rules. Our result strongly supports ability to pay doctrine from the view point of strategic behavior. First we show that effective strategy-proofness implies nonbossiness.

Proposition 1. Consider the public good economy with a private good and a public good where the domain is classical, or it is quasi-linear. If a rule is effectively pairwise strategy-proof, then it is nonbossy.

Proposition 1 holds even without resource-balance ${ }^{12}$. It is a special feature of the type of economies analyzed in this section that effective pairwise strategy-proofness alone implies nonbossiness.

Theorem 1. Consider the public good economy with a private good and a public good where

\footnotetext{
${ }^{12} \mathrm{An}$ allocation is resource-balanced if all endowments are used up for the production of other goods or consumed by some agents. A rule $f$ is resource-balanced if for any $u \in U, f(u)$ is resource-balanced.
} 
the domain is classical, or it is quasi-linear. If a rule is effectively pairwise strategy-proof, then it is a preference independent cost share rule.

Theorem 1 implies that to be a preference independent cost share rule is a necessary condition of effective pairwise strategy-proofness. However, to be a preference independent cost share rule is not a sufficient condition of effective pairwise strategy-proofness, as illustrated by Example 2 below.

Example 2. Assume that $n=3$ and that $C(y)=y$ for any $y \in \mathbb{R}_{+}$. Let $t_{1}$ be such that $t_{1}(y)=y / 2$ for any $y \in[0,2]$ and $t_{1}(y)=1+(y-2) / n$ for any $y>2$. For each $i \in N \backslash\{1\}$, let $t_{i}(y)=\left[y-t_{1}(y)\right] /(n-1)$ for any $y \in \mathbb{R}_{+}$. When the level of the public good is determined similarly to Example $1^{13}$, the rule is not even strategy-proof.

Example 3. Assume that $C, t_{1}$, and $t_{i}(y)$ for all $i \in N \backslash\{1\}$ are the same as Example 2. If the public good is always produced as much as the demand of some prespecified agent, for instance, if the public good is always produced as much as agent 2's demand, then the rule is group strategy-proof.

Whether a preference independent cost share rule satisfies strategy-proof requirements or not depends on several factors. Examples 1, 2, and 3 imply that the shape of the norm cost share functions, and the power of structure to decide the level of the public good are important. It is well-known that if the production range, $Y_{f}$, is convex, if the norm cost functions are all convex, and if the public good production level is determined by the median voter scheme, then the rule is group strategy-proof. Moulin (1980) generalizes the median voter scheme, and identifies the class of strategy-proof schemes determining public good production levels. ${ }^{14}$ Serizawa $(1996,1999)$ and Ohseto $(1997)$ provide various sufficient conditions for strategy-proof requirements.

Effective pairwise strategy-proofness is actually a strong condition in public goods economies. It is equivalent to group strategy-proofness on the class of the classical preferences, as Proposition 2 below says. However, it is an open question whether the same equivalence holds or not on the class of quasi-linear preferences.

Proposition 2. Consider the public good economy with a private good and a public good where the domain is classical. A rule is effectively pairwise strategy-proof if and only if it is group strategy-proof.

\section{Pure Exchange Economies}

In this section, we consider pure exchange economies, that is, economies where there are only private goods, and there is no production. Thus, $m_{Y}=0$ in this section. The feasible set $Z$ in this section is the set $\left\{z=\left(x^{1}, \ldots, x^{n}\right) \in X^{1} \times \cdots \times X^{n} \mid \sum_{i \in N} x^{i} \leq \sum_{i \in N} e^{i}\right\}$.

Definition. A preference $u^{i} \in U^{0}$ is homothetic if for any $z^{i} \in Z^{i}$, any $\widehat{z}^{i} \in Z^{i}$, and $\lambda$ $\in \mathbb{R}_{+}$,

$$
u^{i}\left(z^{i}\right) \geq u^{i}\left(\widehat{z}^{i}\right) \Rightarrow u^{i}\left(\lambda \cdot z^{i}\right) \geq u^{i}\left(\lambda \cdot \widehat{z}^{i}\right) .
$$

\footnotetext{
${ }^{13}$ The preferences of agents, $j=2, \ldots, n$, induce single-peaked preferences on $Y_{f}$. However, the preference on $Y_{f}$ induced by agent 1's preference may have two peaks. In that case, we take the larger one as agent 1's demand, for example.

${ }^{14}$ Barberà, Massó, and Serizawa (1998) also study the case where there are several public goods and the production range is compact.
} 
We denote the class of homothetic preferences by $U^{H}$, and call it the homothetic domain.

Definition. A preference $u^{i} \in U^{0}$ is smooth if for any $z^{i} \in \mathbb{R}_{++}^{m}$, there is a unique vector in the unit simplex $\left\{q \in \mathbb{R}_{+}^{m}:\|q\|=1\right\}$ that generates a hyperplane supporting $U C\left(u^{i}, z^{i}\right)$ at $z^{i}$.

We denote the class of smooth preferences by $U^{M}$, and call it the smooth domain.

In his pioneering article, Hurwicz (1972) establishes that there is no strategy-proof, Pareto-efficient, and individually rational rule for pure exchange economies with two agents and two goods, provided that the domain includes a sufficiently wide class of classical preferences. However, he left open the many-agent case. By citing the following example from Satterthwaite and Sonnenschein (1981), Zhou (1991) later demonstrates that the results of the two-agent case may not extend to the many-agent case.

Example 4 (Satterthwaite and Sonnenschein, 1981). Suppose that there are two goods and three agents, say, agents 1, 2, and 3. Agent 1 gets the whole endowment if agent 3's marginal rate of substitution at $(1,1)$ is greater than 1 . Otherwise, agent 2 gets the whole endowment.

The rule of Example 4 is strategy-proof, Pareto-efficient, and nondictatorial, although Zhou (1991) proves the non-existence of such a rule in the two-agent case. He establishes that in pure exchange economies with two agents and any finite number of goods, there is no strategy-proof, Pareto-efficient, and nondictatorial rule on the class of classical preferences. Schummer (1997) establishes the same conclusion as Zhou (1991) on the class of classical and homothetic preferences in the two-agent case. Serizawa (2002) has recently shown the parallel result of Hurwicz (1972) on the class of classical, homothetic, and smooth preferences in the case of any finite number of agents and goods. Furthermore, Serizawa and Weymark (2003) strengthen Serizawa's (2002) result. They replace individual rationality by a weaker condition of "minimum consumption guarantee"; it requires that there should be a positive constant $\epsilon$ such that for any preference profile, the consumption of every agent is at least $\epsilon$ distance from the origin of his consumption set. 15

By employing effective pairwise strategy-proofness, we establish a new result for pure exchange economies with any finite number of agents and goods. We show that in pure exchange economies with any finite number of agents and goods, there is no effectively pairwise strategy-proof, Pareto-efficient, and nondictatorial rule on the class of classical, homothetic and smooth preferences. When compared with Hurwicz (1972), Zhou (1991) and Schummer (1997), our result can be applied to pure exchange economies with any finite number of agents. When compared with Serizawa (2002) and Serizawa and Weymark (2003), individual rationality and minimum consumption guarantee are replaced with the weaker condition of nondictatoriality. First we show that effective pairwise strategyproofness and Pareto-efficiency imply nonbossiness.

Proposition 3. Consider the pure exchange economy where there are at least two private goods and two agents, and the domain is classical, homothetic and smooth. If a rule is

\footnotetext{
${ }^{15}$ To be precise, individual rationality implies minimum consumption guarantee in the case that every agent has positive endowments of all goods. Otherwise, the two requirements are mathematically independent.
} 
effectively pairwise strategy-proof and Pareto-efficient, then it is nonbossy.

We emphasize that in pure exchange economies, effective pairwise strategy-proofness and nonbossiness are mathematically independent. Satterthwaite and Sonnenschein (1981) explain that a Walrasian rule is nonbossy, but it is not even strategy-proof. Example 5 below illustrates that pairwise strategy-proofness alone does not imply nonbossiness.

Example 5. Consider a pure exchange economy with $N=\{1,2,3\}, M=\{1,2\}$, and $e^{1}=e^{2}=e^{3}=(2,2)$. Assume that the domain is classical, homothetic and smooth. Let $A=\{(1,3),(2,2),(3,1)\}$. Given $u \in U$, let $B^{i}(u)$ be the set of agent $i$ 's best elements on $A$. The difference between $B^{i}(u)$ and $e^{i}$ is considered to be agent $i^{\prime}$ s (net) demand or supply. Note that $\# B^{i}(u)=1$ or $\# B^{i}(u)=2 .{ }^{16}$ Also note that if $\# B^{i}(u)=2$, then $B^{i}(u)=\{(1,3),(2,2)\}$ or $\{(2,2),(3,1)\}$. We construct the "fixed price trading between agents 1 and 2" as follows: We set $f^{3}(u)=e^{3}=(2,2)$ for any $u \in U$. We fix the relative price between goods 1 and 2 to be 1 so that the trades between agents 1 and 2 are constrained to $A$, that is, $f(U) \subseteq A \times A \times\left\{e^{3}\right\}$. We choose the allocation depending on the number of the agents whose $B^{i}$ consist of two elements, $\#\left\{i \in N: \# B^{i}(u)=2\right\}$.

Case I: $\# B^{1}(u)=\# B^{2}(u)=1$. "The short side principle" is employed; it says that the goods are traded as much as the smaller side of demand or supply. For example, if $B^{1}(u)=\{(1,3)\}$ and $B^{2}(u)=\{(3,1)\}$, then $f(u)=((1,3),(3,1),(2,2))$. Or if $B^{1}(u)=$ $\{(1,3)\}$ and $B^{2}(u)=\{(2,2)\}$, then $f(u)=((2,2),(2,2),(2,2))$.

Case II: $\left[\# B^{1}(u)=1 \& \# B^{2}(u)=2\right]$ or $\left[\# B^{1}(u)=2 \& \# B^{2}(u)=1\right]$. The tie of $B^{i}$ with $\# B^{i}(u)=2$ is broken in the way to minimize the difference between supply and demand; and then the short side principle is applied. For example, if $B^{1}(u)=$ $\{(1,3),(2,2)\}$ and $B^{2}(u)=\{(3,1)\}$, then $f(u)=((1,3),(3,1),(2,2))$. Or if $B^{1}(u)=$ $\{(1,3),(2,2)\}$ and $B^{2}(u)=\{(2,2)\}$, then $f(u)=((2,2),(2,2),(2,2))$.

Case III: $\left[\# B^{1}(u)=\# B^{2}(u)=2\right]$. Case III is divided into two subcases.

Subcase III-i: $\left[B^{1}(u)=B^{2}(u)=\{(1,3),(2,2)\}\right]$ or $\left[B^{1}(u)=B^{2}(u)=\{(3,1),(2,2)\}\right]$ or $\left[B^{1}(u)=\{(1,3),(2,2)\} \& B^{2}(u)=\{(3,1),(2,2)\}\right]$. We choose $f(u)=((2,2),(2,2),(2,2))$ for any such preference profile $u \in U$.

Subcase III-ii: $\left[B^{1}(u)=\{(3,1),(2,2)\} \& B^{2}(u)=\{(1,3),(2,2)\}\right]$. In this subcase, we use agent 3 's preference to choose the allocation. We set $f(u)=((3,1),(1,3),(2,2))$ if $u^{3}(1,3) \geq u^{3}(3,1)$, and set $f(u)=((2,2),(2,2),(2,2))$ otherwise.

Then $f$ is pairwise strategy-proof, and even group strategy-proof, but it is bossy in Subcase III-ii.

Now we state the main result of this section.

Theorem 2. Consider the pure exchange economy where there are at least two private goods and at least two agents, and the domain is classical, homothetic and smooth. There is no effectively pairwise strategy-proof, Pareto-efficient, and nondictatorial rule.

Corollary. Consider the pure exchange economy where there are at least two private goods and at least two agents. Let the domain $U$ be a superset of the class of classical, homothetic and smooth preferences. Then there is no effectively pairwise strategy-proof, Pareto-efficient, and nondictatorial rule on $U$.

Proposition 4 also follows from Theorem 2 since dictatorships are group strategy-proof.

\footnotetext{
${ }^{16}$ Given a set $D$, we denote the cardinality of $D$ by $\# D$.
} 
Proposition 4. Consider the pure exchange economy where there are at least two private goods and two agents, and the domain is classical, homothetic and smooth. If a rule is effectively pairwise strategy-proof and Pareto-efficient, then it is group strategy-proof.

Remember the rule of Example 4. This rule is strategy-proof and Pareto-efficient, but it is not effectively pairwise strategy-proof. Thus strategy-proofness and Pareto-efficiency do not imply effective pairwise strategy-proofness. However, it is an open question whether effective pairwise strategy-proofness alone implies group strategy-proofness or not in pure exchange economies.

\section{Allotment Economies}

In this section, we consider the following allotment economies: There is only one private good that is not freely disposable; agents have single-peaked preferences; and the total endowment of the good is distributed among agents. Thus, in this section, $m_{X}=1$ and $m_{Y}=0$, and the feasible set $Z$ is the set $\left\{z=\left(x^{1}, \ldots, x^{n}\right) \in \mathbb{R}_{+}^{n} \mid \sum_{i \in N} x^{i}=\Omega\right\}$, where $\Omega$ is the total endowment of the private good.

Definition. A preference $u^{i} \in U^{0}$ on $Z^{i}=\mathbb{R}_{+}$is single-peaked if it has a unique best element $b\left(u^{i}\right)$ (the "peak" of $u^{i}$ ), and for any $z^{i} \in \mathbb{R}_{+}$and any $\widehat{z}^{i} \in \mathbb{R}_{+}$,

$$
\left[z^{i}<\widehat{z}^{i} \leq b\left(u^{i}\right) \text { or } z^{i}>\widehat{z}^{i} \geq b\left(u^{i}\right)\right] \Longrightarrow u^{i}\left(\widehat{z}^{i}\right)>u^{i}\left(z^{i}\right) \text {. }
$$

We denote the class of single-peaked preferences by $U^{S}$, and call it the single-peaked domain.

Throughout this section, we assume that the domain is single-peaked. Sprumont (1991) cites several interesting economic situations, which induce allotment economies. In this type of economies, he characterizes the "uniform rule (Benassy, 1982)" defined below; he establishes that the uniform rule is the only rule that is strategy-proof, anonymous, and Pareto-efficient. Later Ching (1994) strengthens this result by substituting anonymity by symmetry. That is, he establishes that the uniform rule is the only rule that is strategy-proof, symmetry, and Pareto-efficient. When effective pairwise strategyproofness is applied to allotment economies, a new characterization of the uniform rule obtains. In the characterization results above, if effective pairwise strategy-proofness is employed instead of strategy-proofness, then Pareto-efficiency can be replaced by respect for unanimity, a much weaker requirement.

Definition. A rule $f$ on $U=\left(U^{S}\right)^{n}$ respects unanimity if for any $u \in U$, whenever $\sum_{i \in N} b\left(u^{i}\right)=\sum_{i \in N} e^{i}, f(u)=\left(b\left(u^{i}\right)\right)_{i \in N}$.

We establish that the uniform rule is the only rule that is effectively pairwise strategyproof and symmetric, and respects unanimity. We also establish that effective pairwise strategy-proofness together with respect for unanimity imply group strategy-proofness.

The uniform rule allocates the total endowment to agents as follows. When the sum of agents' preferred levels is greater than or equal to the total endowment, an agent gets his preferred level if that level is less than the common upper bound; otherwise he receives the common bound; and the common bound is chosen so as to satisfy the feasibility. When the sum of agents' preferred levels is less than the total endowment, the opposite principle is applied, that is, an agent gets his preferred level if that level is more than the common lower bound; and so on. 
Definition. The uniform rule $F$ is the function from $U=\left(U^{S}\right)^{n}$ to $Z$ such that for all $u \in U=\left(U^{S}\right)^{n}$ and all $i \in N$,

$$
F^{i}(u)= \begin{cases}\min \left\{b\left(u^{i}\right), \lambda(u)\right\} & \text { if } \Omega \leq \sum_{i \in N} b\left(u^{j}\right) \\ \max \left\{b\left(u^{i}\right), \lambda(u)\right\} & \text { otherwise }\end{cases}
$$

where $\lambda(u)$ solves $\sum_{i \in N} F^{i}(u)=\Omega$.

Proposition 5. If a rule in an allotment economy is effectively pairwise strategy-proof and respects unanimity, then it is nonbossy.

Remember Example 5 of fixed price trading in Section 4. As Sprumont (1991) discusses, fixed price trading induces allotment economies. Since the trades of agents are constrained to $A$, the rule in the example does not respect unanimity. Thus Example 5 also demonstrates that effective pairwise strategy-proofness alone does not imply nonbossiness in allotment economies.

Proposition 6. If a rule in an allotment economy is effectively pairwise strategy-proof and respects unanimity, then it is Pareto-efficient.

Theorem 3 below follows from Proposition 6, and Sprumont (1992) and Ching (1994).

Theorem 3. A rule in an allotment economy is effectively pairwise strategy-proof and symmetric, and respects unanimity if and only if it is the uniform rule.

It is obvious that "only if" Part of Theorem 3 does not hold when any of the three requirements of effective pairwise strategy-proofness, symmetry, and respect for unanimity is dropped. The "proportional rule" defined below satisfies symmetry and respect for unanimity, but not effective pairwise strategy-proofness. The "queuing rule" defined below satisfies pairwise strategy-proofness and respect for unanimity, but not symmetry. The "equal distribution rule" satisfies pairwise strategy-proofness and symmetry, but not respect for unanimity. However, it is an open question whether the uniform rule is the unique rule that satisfies individual strategy-proofness, symmetry, and respect for unanimity.

Definition. The proportional rule $P$ is the function from $U=\left(U^{S}\right)^{n}$ to $Z$ such that for all $u \in U=\left(U^{S}\right)^{n}$ and all $i \in N, P^{i}(u)=\left(\Omega \cdot b\left(u^{i}\right)\right) /\left(\sum_{j \in N} b\left(u^{j}\right)\right)$.

Definition. The queuing rule $Q$ is the function from $U=\left(U^{S}\right)^{n}$ to $Z$ such that there is a permutation $\pi$ of $N$ and for all $u \in U=\left(U^{S}\right)^{n}$,

$$
\begin{aligned}
& Q^{\pi(1)}(u)=\operatorname{Argmax}\left\{u^{\pi(1)}\left(z^{0}\right): z^{0} \in[0, \Omega]\right\}, \\
& Q^{\pi(2)}(u)=\operatorname{Argmax}\left\{u^{\pi(2)}\left(z^{0}\right): z^{0} \in\left[0, \Omega-Q^{\pi(1)}(u)\right]\right\}, \\
& Q^{\pi(3)}(u)=\operatorname{Argmax}\left\{u^{\pi(3)}\left(z^{0}\right): z^{0} \in\left[0, \Omega-Q^{\pi(1)}(u)-Q^{\pi(2)}(u)\right]\right\}, \\
& \quad \vdots \\
& Q^{\pi(n)}(u)=\Omega-\sum_{j=1}^{j=n-1} Q^{\pi(j)}(u) .
\end{aligned}
$$

Definition. The equal distribution rule $E$ is the function from $U=\left(U^{S}\right)^{n}$ to $Z$ such that for all $u \in U=\left(U^{S}\right)^{n}$ and all $i \in N, E^{i}(u)=\Omega / n$.

Proposition 7. If a rule in an allotment economy is effectively pairwise strategy-proof and respects unanimity, then it is group strategy-proof. 
Strategy-proofness and respect for unanimity do not imply group strategy-proofness, as Example 6 shows.

Example 6. Let $N=\{1,2,3\}$. The following rule $f$ is a variant of the queuing rule. Let $u \in$ $U$ be given. $f^{1}(u)=b\left(u^{1}\right)$. If $u^{1}(\Omega)>u^{1}(0)$, then $f^{2}(u)=\operatorname{Argmax}\left\{u^{2}\left(z^{0}\right): z^{0} \in[0, \Omega-\right.$ $\left.\left.f^{1}(u)\right]\right\}$ and $f^{3}(u)=\Omega-f^{1}(u)-f^{2}(u)$. If $u^{1}(\Omega) \leq u^{1}(0)$, then $f^{3}(u)=\operatorname{Argmax}\left\{u^{3}\left(z^{0}\right)\right.$ : $\left.z^{0} \in\left[0, \Omega-f^{1}(u)\right]\right\}$ and $f^{2}(u)=\Omega-f^{1}(u)-f^{3}(u)$. Then $f$ is not effectively pairwise strategy-proof or group strategy-proof, but it is strategy-proof and respects unanimity.

\section{Proofs}

In this section, we prove the results in Sections 3, 4, and 5. Subsection 6.1 introduces a well-known notion of "Maskin Monotonic Transformation (Maskin, 1999)," and shows the basic results of effective pairwise strategy-proofness. Subsections 6.2, 6.3 and 6.4 are respectively devoted to the proofs of the results in Sections 3, 4, and 5 .

\subsection{Preliminary Results}

Given $z^{i} \in Z^{i}$ and $u^{i} \in U^{0}$, let the upper contour set $U C\left(u^{i}, z^{i}\right)=\left\{\widehat{z}^{i} \in Z^{i} \mid u^{i}\left(\widehat{z}^{i}\right) \geq\right.$ $\left.u^{i}\left(z^{i}\right)\right\}$, and the lower contour set $L C\left(u^{i}, z^{i}\right)=\left\{\widehat{z}^{i} \in Z^{i} \mid u^{i}\left(\widehat{z}^{i}\right) \leq u^{i}\left(z^{i}\right)\right\}$. We say that $\hat{u}^{i} \in U^{0}$ is a Maskin Monotonic Transformation ${ }^{17}$ of $u^{i}$ at $z^{i}$ if (i) $U C\left(\hat{u}^{i}, z^{i}\right) \subseteq U C\left(u^{i}, z^{i}\right)$ holds, and (ii) $\widehat{z}^{i} \in U C\left(\hat{u}^{i}, z^{i}\right)$ and $\widehat{z}^{i} \neq z^{i}$ together imply that $u^{i}\left(\widehat{z}^{i}\right)>u^{i}\left(z^{i}\right)$. Let $M\left(u^{i}, z^{i}\right)$ be the set of Maskin Monotonic Transformations of $u^{i}$ at $z^{i}$. Given $u^{i} \in U^{0}$ and $z \in Z$, we write $U C\left(u^{i}, z\right)=U C\left(u^{i}, z^{i}\right)$ and $M\left(u^{i}, z\right)=M\left(u^{i}, z^{i}\right)$ when it simplifies notation without creating confusion. Fact 1 below is a well-known result. ${ }^{18}$

Fact 1. Let $f$ be a strategy-proof rule. For any $u \in U$, any $i \in N$, and any $\hat{u}^{i} \in$ $M\left(u^{i}, f(u)\right), f^{i}\left(\hat{u}^{i}, u^{-i}\right)=f^{i}(u)$.

Lemma 1.1. Let $f$ be an effectively pairwise strategy-proof rule. Let $u \in U, i \in N$, and $\hat{u}^{i} \in U^{i}$ be such that $u^{i}\left(f^{i}\left(\hat{u}^{i}, u^{-i}\right)\right)=u^{i}\left(f^{i}(u)\right)$ and $\hat{u}^{i}\left(f^{i}\left(\hat{u}^{i}, u^{-i}\right)\right)=\hat{u}^{i}\left(f^{i}(u)\right)$. Then for any $j \neq i, u^{j}\left(f^{j}\left(\hat{u}^{i}, u^{-i}\right)\right)=u^{j}\left(f^{j}(u)\right)$.

Proof of Lemma 1.1. Let $j \neq i$. First, we prove $u^{j}\left(f^{j}\left(\hat{u}^{i}, u^{-i}\right)\right) \leq u^{j}\left(f^{j}(u)\right)$. Suppose $u^{j}\left(f^{j}\left(\hat{u}^{i}, u^{-i}\right)\right)>u^{j}\left(f^{j}(u)\right)$. Then $\left(\hat{u}^{i}, u^{j}\right)$ is a self-enforcing manipulation of the pair $N^{\prime}=\{i, j\}$ at the preference profile $u$. To see this, note that if it is not, Condition (iii) or (iv) of effective pairwise strategy-proofness must be violated. If Condition (iii) is violated, $u^{i}\left(f^{i}\left(\widetilde{u}^{i}, u^{-i}\right)\right)>u^{i}\left(f^{i}\left(\hat{u}^{i}, u^{-i}\right)\right)$ for some $\widetilde{u}^{i} \in U^{i}$. But, since $u^{i}\left(f^{i}\left(\hat{u}^{i}, u^{-i}\right)\right)=u^{i}\left(f^{i}(u)\right)$, this contradicts to individual strategy-proofness for agent $i$. If Condition (iv) is violated, $u^{j}\left(f^{j}\left(\widetilde{u}^{j}, \hat{u}^{i}, u^{-N^{\prime}}\right)\right)>u^{j}\left(f^{j}\left(\hat{u}^{i}, u^{-i}\right)\right)$ for some $\widetilde{u}^{j} \in U^{j}$. This also contradicts to individual strategy-proofness for agent $j$. Therefore, $u^{j}\left(f^{j}\left(\hat{u}^{i}, u^{-i}\right)\right) \leq u^{j}\left(f^{j}(u)\right)$.

If $u^{j}\left(f^{j}\left(\hat{u}^{i}, u^{-i}\right)\right)<u^{j}\left(f^{j}(u)\right)$, by the same way as above, we can show that $\left(u^{i}, u^{j}\right)$ is a self-enforcing manipulation of the pair $N^{\prime}=\{i, j\}$ at the preference profile $\left(\hat{u}^{i}, u^{-i}\right)$, which is a contradiction Therefore, $u^{j}\left(f^{j}\left(\hat{u}^{i}, u^{-i}\right)\right) \geq u^{j}\left(f^{j}(u)\right)$. Q.E.D.

Lemma 1.2 below directly follows from Lemma 1.1.

\footnotetext{
${ }^{17}$ To be precise, this is the definition of what is called "Strictly Maskin Monotonic Transformation". Here, we call this notion "Maskin Monotonic Transformation" for short.

${ }^{18}$ See, for example, Zhou (1991), Barberà and Jackson (1995), etc.
} 
Lemma 1.2. Let $f$ be an effectively pairwise strategy-proof rule. Let $u \in U, i \in N$, and $\hat{u}^{i} \in U^{i}$ be such that $f^{i}\left(\hat{u}^{i}, u^{-i}\right)=f^{i}(u)$. Then, for any $j \neq i, u^{j}\left(f^{j}\left(\hat{u}^{i}, u^{-i}\right)\right)=u^{j}\left(f^{j}(u)\right)$.

Lemma 1.3 below follows from Fact 1 and Lemma 1.2.

Lemma 1.3. Let $f$ be an effectively pairwise strategy-proof rule. For any $u \in U$, any $i \in N$, any $\hat{u}^{i} \in M\left(u^{i}, f(u)\right)$, and any $j \neq i, u^{j}\left(f^{j}\left(\hat{u}^{i}, u^{-i}\right)\right)=u^{j}\left(f^{j}(u)\right)$.

Proof of Lemma 1.3. Let $u \in U, i \in N, \hat{u}^{i} \in M\left(u^{i}, f(u)\right)$, and $j \neq i$. Since $f^{i}\left(\hat{u}^{i}, u^{-i}\right)=$ $f^{i}(u)$ by Fact 1, Lemma 1.2 implies $u^{j}\left(f^{j}\left(\hat{u}^{i}, u^{-i}\right)\right)=u^{j}\left(f^{j}(u)\right)$. Q.E.D.

\subsection{Proofs of Results in Section 3}

In this subsection, we prove the results in Section 3.

Proof of Proposition 1. Let $f$ be an effectively pairwise strategy-proof rule, and let $U=U^{1} \times \cdots \times U^{n}$ be the domain. The following argument holds whether $U$ is classical or $U$ is quasi-linear and separable. Let $u \in U, i \in N$, and $\hat{u}^{i} \in U^{i}$ be such that $f^{i}(u)=$ $f^{i}\left(\hat{u}^{i}, u^{-i}\right)$. We want to show that $f^{-i}(u)=f^{-i}\left(\hat{u}^{i}, u^{-i}\right)$. Suppose $f^{-i}(u) \neq f^{-i}\left(\hat{u}^{i}, u^{-i}\right)$. Since $f^{i}(u)=f^{i}\left(\hat{u}^{i}, u^{-i}\right)$ implies $f_{y}(u)=f_{y}\left(\hat{u}^{i}, u^{-i}\right)$, there is $j \in N$ such that $f_{j}(u) \neq$ $f_{j}\left(\hat{u}^{i}, u^{-i}\right)$. If $f_{j}(u)<f_{j}\left(\hat{u}^{i}, u^{-i}\right)$, then $u^{j}(f(u))<u^{j}\left(f\left(\hat{u}^{i}, u^{-i}\right)\right.$. If $f_{j}(u)>f_{j}\left(\hat{u}^{i}, u^{-i}\right)$, then $u^{j}(f(u))>u^{j}\left(f\left(\hat{u}^{i}, u^{-i}\right)\right.$. This contradicts Lemma 1.2. Q.E.D.

Proof of Theorem 1. The following argument holds whether the domain $U$ is classical, or $U$ is quasi-linear. Suppose that a rule $f$ is not a preference independent cost share rule. Then there are $u \in U$ and $\widehat{u} \in U$ such that $f_{y}(u)=f_{y}(\widehat{u})$ but $f_{i}(u) \neq f_{i}(\widehat{u})$ for some $i \in N$. Since $f_{y}(u)=f_{y}(\widehat{u})$, for each agent $j \in N$, there is $\widetilde{u}^{j} \in M\left(u^{j}, f(u)\right) \cap M($ $\left.\widehat{u}^{j}, f(\widehat{u})\right)$. By Fact 1 and nonbossiness (Proposition 1), we have that $f(u)=f(\widetilde{u})=f(\widehat{u})$, which is a contradiction. Q.E.D.

Proof of Proposition 2. Since "If" Part is trivial, we show only "Only if" Part. Let $f$ be an effectively pairwise strategy-proof rule. Suppose that $f$ is not group strategy-proof. We derive a contradiction. Note that there are $u \in N, N^{\prime}=C_{1} \cup C_{2} \subseteq N$, and $\widehat{u}^{N^{\prime}} \in U^{N^{\prime}}$ such that

(1) for any $i \in C_{1}, u^{i}\left(f\left(\widehat{u}^{N^{\prime}}, u^{-N^{\prime}}\right)\right)=u^{i}(f(u))$;

(2) for any $i \in C_{2}, u^{i}\left(f\left(\widehat{u}^{N^{\prime}}, u^{-N^{\prime}}\right)\right)>u^{i}(f(u))$; and

(3) $C_{2} \neq \emptyset$.

Let $z=f(u)$ and $\widehat{z}=f\left(\widehat{u}^{N^{\prime}}, u^{-N^{\prime}}\right)$. Since the domain is classical, by (2), for each $i \in C_{2}$, there is $\widetilde{u}^{i} \in M\left(u^{i}, z\right) \cap M\left(\widehat{u}^{i}, \widehat{z}\right)$. Then, since $z=f(u)$, by applying Fact 1 successively for agents in $C_{2}$ and nonbossiness (Proposition 1), we have (4) $f\left(u^{C_{1}}, \widetilde{u}^{C_{2}}, u^{-N^{\prime}}\right)=z$. Similarly, we have (5) $f\left(\widehat{u}^{C_{1}}, \widetilde{u}^{C_{2}}, u^{-N^{\prime}}\right)=\widehat{z}$. If $C_{1}=\emptyset$, since (2) and (3) imply $\widehat{z} \neq z$, we already have a contradiction. Thus we assume $C_{1} \neq \emptyset$. Without loss of generality, let $C_{1}=\{1, \ldots, c\}$.

Note that since the domain is classical, given $\epsilon>0$, there is $\widetilde{u}_{\epsilon}^{1} \in M\left(\widehat{u}^{1}, \widehat{z}^{1}\right) \cap M\left(u^{1}, \widehat{z}^{1}\right)$ such that

$$
\forall z^{1 \prime} \in Z^{1}:\left[z^{1 \prime} \in U C\left(\widetilde{u}_{\epsilon}^{1}, z^{1}\right) \&\left\|z^{1 \prime}-\widehat{z}^{1}\right\|>\epsilon \& z^{1 \prime} \neq z^{1}\right] \Rightarrow u^{1}\left(z^{1 \prime}\right)>u^{1}\left(z^{1}\right) .
$$

Figure 1 below illustrates the preference $\widetilde{u}_{\epsilon}^{1}$. Note that by $(1), \widetilde{u}_{\epsilon}^{1} \in M\left(u^{1}, \widehat{z}^{1}\right)$ implies that $(7) \widetilde{u}_{\epsilon}^{1}\left(\widehat{z}^{1}\right)>\widetilde{u}_{\epsilon}^{1}\left(z^{1 \prime}\right)$ for any $z^{1 \prime} \in L C\left(u^{1}, z^{1}\right) \backslash\left\{\widehat{z}^{1}\right\}$.

In this paragraph, we claim that for some $\epsilon>0, f^{1}\left(\widetilde{u}_{\epsilon}^{1}, u^{\{2, \ldots, c\}}, \widetilde{u}^{C_{2}}, u^{-N^{\prime}}\right)=z^{1}$ or $f^{1}\left(\widetilde{u}_{\epsilon}^{1}, u^{\{2, \ldots, c\}}, \widetilde{u}^{C_{2}}, u^{-N^{\prime}}\right)=\widehat{z}^{1}$. Suppose not. Then, there is a sequence $\{\epsilon(k)\}_{k=1}^{k=\infty}$ such 
that $(8) \epsilon(k) \rightarrow 0$ as $k \rightarrow \infty$; and (9) for any $k, f^{1}\left(\widetilde{u}_{\epsilon(k)}^{1}, u^{\{2, \ldots, c\}}, \widetilde{u}^{C_{2}}, u^{-N^{\prime}}\right) \neq z^{1}$ and $f^{1}\left(\widetilde{u}_{\epsilon(k)}^{1}, u^{\{2, \ldots, c\}}, \widetilde{u}^{C_{2}}, u^{-N^{\prime}}\right) \neq \widehat{z}^{1}$. Note that by (4), strategy-proofness implies that (10) $f^{1}\left(\widetilde{u}_{\epsilon(k)}^{1}, u^{\{2, \ldots, c\}}, \widetilde{u}^{C_{2}}, u^{-N^{\prime}}\right) \in U C\left(\widetilde{u}_{\epsilon(k)}^{1}, z^{1}\right) \cap L C\left(u^{1}, z^{1}\right)$ for any $k$. Note further that by (6) and (8), the set $\left[U C\left(\widetilde{u}_{\epsilon(k)}^{1}, z^{1}\right) \cap L C\left(u^{1}, z^{1}\right)\right] \backslash\left\{z^{1}\right\}$ converges to $\left\{\widehat{z}^{1}\right\}$ as $k \rightarrow \infty$. Thus, it follows from (9) and (10) that $f^{1}\left(\widetilde{u}_{\epsilon(k)}^{1}, u^{\{2, \ldots, c\}}, \widetilde{u}^{C_{2}}, u^{-N^{\prime}}\right) \rightarrow \widehat{z}^{1}$ as $k \rightarrow \infty$. Therefore, it also follows from (7) and (9) that given $k$, there is $K$ such that

$$
\widetilde{u}_{\epsilon(k)}^{1}\left(f^{1}\left(\widetilde{u}_{\epsilon(K)}^{1}, u^{\{2, \ldots, c\}}, \widetilde{u}^{C_{2}}, u^{-N^{\prime}}\right)\right)>\widetilde{u}_{\epsilon(k)}^{1}\left(f^{1}\left(\widetilde{u}_{\epsilon(k)}^{1}, u^{\{2, \ldots, c\}}, \widetilde{u}^{C_{2}}, u^{-N^{\prime}}\right)\right),
$$

contradicting strategy-proofness for agent 1. Accordingly, for some $\epsilon>0$, we have $f\left(\widetilde{u}_{\epsilon}^{1}, u^{\{2, \ldots, c\}}, \widetilde{u}^{C_{2}}, u^{-N^{\prime}}\right)=z^{1}$ or $f^{1}\left(\widetilde{u}_{\epsilon}^{1}, u^{\{2, \ldots, c\}}, \widetilde{u}^{C_{2}}, u^{-N^{\prime}}\right)=\widehat{z}^{1}$.

Let $\epsilon_{1}>0$ be such that $f^{1}\left(\widetilde{u}_{\epsilon_{1}}^{1}, u^{\{2, \ldots, c\}}, \widetilde{u}^{C_{2}}, u^{-N^{\prime}}\right)=z^{1}$ or $f^{1}\left(\widetilde{u}_{\epsilon_{1}}^{1}, u^{\{2, \ldots, c\}}, \widetilde{u}^{C_{2}}, u^{-N^{\prime}}\right)=$ $\widehat{z}^{1}$. If $f^{1}\left(\widetilde{u}_{\epsilon_{1}}^{1}, u^{\{2, \ldots, c\}}, \widetilde{u}^{C_{2}}, u^{-N^{\prime}}\right)=\widehat{z}^{1}$, then by Theorem 1, $f\left(\widetilde{u}_{\epsilon_{1}}^{1}, u^{\{2, \ldots, c\}}, \widetilde{u}^{C_{2}}, u^{-N^{\prime}}\right)=$ $\widehat{z}$. By (1), (2), (3), and (4), $\left(\widetilde{u}_{\epsilon_{1}}^{1}, \widetilde{u}^{c+1}\right)$ is a self-forcing manipulation by the pair of agents 1 and $(c+1)$ at the profile $\left(u^{C_{1}}, \widetilde{u}^{C_{2}}, u^{-N^{\prime}}\right) .{ }^{19}$ This contradicts effective pairwise strategy-proofness. Thus $f^{1}\left(\widetilde{u}_{\epsilon_{1}}^{1}, u^{\{2, \ldots, c\}}, \widetilde{u}^{C_{2}}, u^{-N^{\prime}}\right)=z^{1}$, and so by Theorem 1 , we have $f\left(\widetilde{u}_{\epsilon_{1}}^{1}, u^{\{2, \ldots, c\}}, \widetilde{u}^{C_{2}}, u^{-N^{\prime}}\right)=z$.

By choosing $\epsilon_{i}$ and $\widetilde{u}_{\epsilon_{i}}^{i}$ similarly for $i=2, \ldots, c$, we obtain $f\left(\widetilde{u}_{\epsilon}^{C_{1}}, \widetilde{u}^{C_{2}}, u^{-N^{\prime}}\right)=z$, where $\widetilde{u}_{\epsilon}^{C_{1}}=\left(\widetilde{u}_{\epsilon_{1}}^{1}, \ldots, \widetilde{u}_{\epsilon_{c}}^{c}\right)$. On the other hand, since $\widetilde{u}_{\epsilon_{i}}^{i} \in M\left(\widehat{u}^{i}, \widehat{z}^{i}\right)$ for each $i \in C_{1}$, it follows from (5), Fact 1, and nonbossiness (Proposition 1) that $f\left(\widetilde{u}_{\epsilon}^{C_{1}}, \widetilde{u}^{C_{2}}, u^{-N^{\prime}}\right)=\widehat{z}$. This is a contradiction. Hence, $f$ is group strategy-proof. Q.E.D.

\section{FIGURE 1 ENTERS HERE.}

\subsection{Proofs of Results in Section 4}

In this subsection, we prove the results in Section 4.

Proof of Proposition 3. Suppose not. Then without loss of generality, we may assume that there are $u \in U$ and $\hat{u}^{1} \in U^{1}$ such that $f^{1}\left(\hat{u}^{1}, u^{-1}\right)=f^{1}(u)$ and $f^{2}\left(\hat{u}^{1}, u^{-1}\right) \neq f^{2}(u)$. Let $f(u)=z$ and $f\left(\hat{u}^{i}, u^{-i}\right)=\hat{z}$. Lemma 1.2 implies that $u^{i}(\hat{z})=u^{i}(z)$ for any $i \in N$. Let $\tilde{z}=(z+\hat{z}) / 2$. Then since preferences are strictly quasi-concave, it follows that $u^{2}(\tilde{z})>u^{2}(z)$ and $u^{j}(\tilde{z}) \geq u^{j}(z)$ for $j \in N$. Because the convexity of $Z$ implies $\tilde{z} \in Z$, this contradicts Pareto-efficiency. Q.E.D.

We introduce several lemmas before we prove Theorem 2.

Lemma 3.1 below says that if a rule is Pareto-efficient and if two agents have identical preferences that are classical and homothetic, then the rule assigns them the proportional bundles. This Lemma is based on the property of classical and homothetic preference that the marginal rate of substitutions at two consumption bundles coincide if and only if the two bundles are proportional. See Schummer (1997) for the proof of the lemma.

Lemma 3.1. Let $f$ be a Pareto-efficient rule, and $u^{0} \in U^{C} \cap U^{H}$. Let $i \in N, j \in N$, and $u \in U$ be such that $u^{i}=u^{j}=u^{0}$. If $f^{i}(u) \neq 0$, then $f^{j}(u)$ is proportional to $f^{i}(u)$, that is, there is $\lambda \in R_{+}$such that $f^{j}(u)=\lambda \cdot f^{i}(u)$.

\footnotetext{
${ }^{19}$ To see that $\left(\widetilde{u}_{\epsilon_{1}}^{1}, \widetilde{u}^{c+1}\right)$ is a self-forcing manipulation, note that Conditions (i) and (ii) of self-forcing manipulation follow from (1), (2), (3), and (4). Conditions (iii) and (iv) follow from strategy-proofness.
} 
Lemma 3.2 below says that if one agent, say agent 1, has classical preferences, and the other agents all have the common preference that is also classical and homothetic, then the locus of the Pareto-efficient allocations looks like a contract curve of a two-agent economy from the viewpoint of agent 1 . To treat the feasible sets and the sets of Pareto-efficient allocations of economies with different number of agents, we introduce new notations. Given $\Omega \in \mathbb{R}_{+}^{m}$ and $n^{\prime} \leq n$, let $Z\left(n^{\prime}, \Omega\right)=\left\{z \in \mathbb{R}_{+}^{m \cdot n^{\prime}} \mid \sum_{i=1}^{n^{\prime}} z^{i}=\Omega\right\}$; that is, $Z\left(n^{\prime}, \Omega\right)$ is the feasible set of the pure exchange economy with the resource bundle $\Omega$ and $n^{\prime}$ agents. Given $\Omega \in \mathbb{R}_{+}^{m}, n^{\prime} \leq n$ and a preference profile $u \in\left(U^{0}\right)^{n^{\prime}}$ for $n^{\prime}$ of agents, denote the set of Pareto-efficient allocations for $u$ on $Z\left(n^{\prime}, \Omega\right)$ by $P\left(u, Z\left(n^{\prime}, \Omega\right)\right)$, and the projection of $P\left(u, Z\left(n^{\prime}, \Omega\right)\right)$ on $Z^{i}$ by $P^{i}\left(u, Z\left(n^{\prime}, \Omega\right)\right)$. Note that the feasible set $Z$ specified in Section 4 coincides with $Z\left(n, \sum_{i \in N} e^{i}\right)$. See Serizawa (2000) for the proof.

Lemma 3.2. Let $\Omega \in R_{+}^{m}, u^{0} \in U^{H}, i \in N$, and $u^{i} \in U^{C}$. Then $P^{i}\left(u^{i}, u^{0}, Z(2, \Omega)\right)=$ $P^{i}\left(u^{i}, u^{0}, \ldots, u^{0}, Z(n, \Omega)\right)$.

Lemma 3.3 is a well known result in literature. For example, it is called "diagonality" in Barberà and Jackson (1995). Thus, we omit its proof.

Lemma 3.3. Let $f$ be a strategy-proof rule on $U$. Let $u \in U$, and $z=f(u)$. Let $i \in N$ and $\widehat{z} \in Z$ be such that $\widehat{z}^{i}<z^{i}$ or $\widehat{z}^{i}>z^{i}$. Then for any $\widehat{u}^{i} \in U^{i}, \widehat{z} \neq f\left(\widehat{u}^{i}, u^{-i}\right)$.

Lemma 3.4. Let $f$ be an effectively pairwise strategy-proof and Pareto-efficient rule on $U$. Let $u^{0} \in U^{C} \cap U^{H}, u^{1} \in U^{C} \cap U^{H}$, and $z=f\left(u^{1}, u^{0}, \ldots, u^{0}\right)$. Let $\widehat{u}^{0} \in M\left(u^{0}, \sum_{i=2}^{i=n} z^{i}\right)$ $\cap U^{C} \cap U^{H}$. Then $z=f\left(u^{1}, \widehat{u}^{0}, \ldots, \widehat{u}^{0}\right)$.

Proof. It follows from Lemma 3.1 that there is $\lambda=\left(\lambda^{2}, \ldots, \lambda^{n}\right) \in \mathbb{R}_{+}^{n-1}$ such that $z^{i}=\lambda^{i} \cdot \sum_{j=2}^{j=n} z^{j}$ for any $i \in N \backslash\{1\}$. Thus since $u^{0}$ and $\widehat{u}^{0}$ are homothetic, for any $i \neq 1$, $\widehat{u}^{0} \in M\left(u^{0}, z^{i}\right)$. Since $\widehat{u}^{0} \in M\left(u^{0}, z^{2}\right)$, Fact 1 implies that $z^{2}=f^{2}\left(u^{1}, \widehat{u}^{0}, u^{0}, \ldots, u^{0}\right)$, and so nonbossiness (Proposition 3) implies $z=f\left(u^{1}, \widehat{u}^{0}, u^{0}, \ldots, u^{0}\right)$. Repeating this argument for $i=3, \ldots, n$, we get the result that $z=f\left(u^{1}, \widehat{u}^{0}, \ldots, \widehat{u}^{0}\right)$. Q.E.D.

In the rest of subsection, $\Omega$ denotes the total endowment, that is, $\Omega=\sum_{i \in N} e^{i}$.

Lemma 3.5. Let $f$ be an effectively pairwise strategy-proof and Pareto-efficient rule on $U$. Let $u^{0} \in U^{C} \cap U^{H}$ and $\widehat{u}^{0} \in U^{C} \cap U^{H}$. Let $z=f\left(u^{0}, \ldots, u^{0}\right)$ and $\widehat{z}=f\left(\widehat{u}^{0}, \ldots, \widehat{u}^{0}\right)$. Then $\widehat{z}=z$.

Proof. By contradiction, suppose that $\widehat{z} \neq z$. Note that by Lemma 3.1 and Paretoefficiency, there are $\lambda=\left(\lambda^{1}, \ldots, \lambda^{n}\right) \in \mathbb{R}_{+}^{n}$ and $\widehat{\lambda}=\left(\widehat{\lambda}^{1}, \ldots, \widehat{\lambda}^{n}\right) \in \mathbb{R}_{+}^{n}$ such that $z=$ $\left(\lambda^{1} \Omega, \ldots, \lambda^{n} \Omega\right)$ and $\widehat{z}=\left(\widehat{\lambda}^{1} \Omega, \ldots, \widehat{\lambda}^{n} \Omega\right)$. Also note that there is $\widetilde{u}^{0} \in U^{C} \cap U^{H}$ such that $\widetilde{u}^{0} \in M\left(u^{0}, \Omega\right) \cap M\left(\widehat{u}^{0}, \Omega\right)$. Then for any $i \in N, \widetilde{u}^{0} \in M\left(u^{0}, z^{i}\right) \cap M\left(\widehat{u}^{0}, \widehat{z}^{i}\right)$. By successively applying Fact 1 and nonbossiness (Proposition 3), we have the result that $z=f\left(\widetilde{u}^{0}, \ldots, \widetilde{u}^{0}\right)$ and $\widehat{z}=f\left(\widetilde{u}^{0}, \ldots, \widetilde{u}^{0}\right)$. This is a contradict. Q.E.D.

Lemma 3.6. Let $f$ be an effectively pairwise strategy-proof, Pareto-efficient, and nondictatorial rule on $U$. There is $d \in Z$ such that $d=\left(\lambda^{1} \Omega, \ldots, \lambda^{n} \Omega\right)$ for some $\lambda=$ $\left(\lambda^{1}, \ldots, \lambda^{n}\right) \in[0,1)^{n}$, and $d=f\left(u^{0}, \ldots, u^{0}\right)$ for any preference $u^{0} \in U^{C} \cap U^{H}$.

Proof. It follows from Lemma 3.5. that there is $d \in Z$ such that $d=\left(\lambda^{1} \Omega, \ldots, \lambda^{n} \Omega\right)$ for some $\lambda=\left(\lambda^{1}, \ldots, \lambda^{n}\right) \in \mathbb{R}_{+}^{n}$, and $d=f\left(u^{0}, \ldots, u^{0}\right)$ for any preference $u^{0} \in U^{C} \cap U^{H}$. Due to the feasibility constraint, for any $i \in N, \lambda^{i} \in[0,1]$. Thus we have only to show that for 
any $i \in N, \lambda^{i} \neq 1$.

By contradiction, suppose that for some $i \in N, \lambda^{i}=1$. Without loss of generality, let $i=1$. We claim that agent 1 is a dictator, that is, for any $u \in U, f(u)=(\Omega, 0, \ldots, 0)$, where $\Omega=\sum_{i=1}^{n} e^{i}$. Let $u \in U$. Let $u^{0} \in U^{C} \cap U^{H}$, and $\widehat{u}=\left(u^{0}, \ldots u^{0}\right)$. Note that $f(\widehat{u})=$ $(\Omega, 0, \ldots, 0)$. Strategy-proofness for agent 1 implies that $f^{1}\left(u^{1}, \widehat{u}^{-1}\right)=\Omega$. For otherwise, $u^{1}(f(\widehat{u}))>u^{1}\left(f\left(u^{1}, \widehat{u}^{-1}\right)\right)$. Thus $f\left(u^{1}, \widehat{u}^{-1}\right)=(\Omega, 0, \ldots, 0)$. Then strategy-proofness for agent 2 implies that $f^{2}\left(u^{1}, u^{2}, \widehat{u}^{-\{1,2\}}\right)=0$. For otherwise, $\widehat{u}^{2}\left(f\left(u^{1}, u^{2}, \widehat{u}^{-\{1,2\}}\right)\right)>$ $\widehat{u}^{2}\left(f\left(u^{1}, \widehat{u}^{-1}\right)\right)$. By nonbossiness (Proposition 3), we have $f\left(u^{1}, u^{2}, \widehat{u}^{-\{1,2\}}\right)=(\Omega, 0, \ldots, 0)$. Repeating this argument for $j=3, \ldots, n$, we get the result that $f(u)=(\Omega, 0, \ldots, 0)$. This is a contradiction to nondictatorship. Q.E.D.

Fact 2 below says that for any classical, homothetic, and smooth preference $u^{0}$ and any consumption bundle $z^{0}$ in the interior of the consumption set, there exists a CES type preference $\widehat{u}^{0}$ that has the same rate of substitution as $u^{0}$ at $z^{0}$ and that is also a Maskin Monotonic Transformation of $u^{0}$.

Fact 2. Let $u^{0}$ be a classical, homothetic, and smooth preference, and let $a \in R_{++}^{m}$ and $z^{0} \in R_{++}^{m}$. Given $\rho \in(-\infty, 1)$ and $x \in R_{+}^{m}$, let $\widehat{u}^{0}(x ; \rho)=A^{-1 / \rho} \cdot\left[\sum_{l \in M} a_{l} \cdot\left(z_{l}^{0}\right)^{1-\rho} \cdot\left(x_{l}\right)^{\rho}\right]^{1 / \rho}$, where $A=\sum_{l \in M} a_{l} \cdot\left(z_{l}^{0}\right)$. Then for any $\rho \in(-\infty, 1)$, the function $\widehat{u}^{0}(\cdot ; \rho)$ on $R_{+}^{m}$ is $a$ classical, homothetic, and smooth preference. If $a_{l}=\left[\partial u^{0}\left(z^{0}\right) / \partial x_{l}\right] \cdot A$ for each $l \in M$, then the following holds:

(i) $\partial \widehat{u}^{0}\left(z^{0} ; \rho\right) / \partial x_{l}=\partial u^{0}\left(z^{0}\right) / \partial x_{l}$ for any $l \in M$.

(ii) There is $\rho_{0} \in(-\infty, 1)$ such that for any $\rho \in\left(-\infty, \rho_{0}\right), \widehat{u}^{0}(\cdot ; \rho) \in M\left(u^{0}, z^{0}\right)$.

Proof of Theorem 2. Let $f$ be an effectively pairwise strategy-proof, Pareto-efficient, and non-dictatorial rule. We apply the above Lemmas to derive a contradiction. Let $d=\left(d^{1}, \ldots, d^{n}\right)=\left(\lambda^{1} \Omega, \ldots, \lambda^{n} \Omega\right)$ be the one specified in Lemma 3.6. Since $\lambda^{i} \in[0,1)$ for any $i \in N$, it follows that there is $j \in N$ such that $\lambda^{j} \in(0,1)$. Without loss of generality, let $j=1$. The basic structure of the rest of the proof is similar to that of the theorem of Schummer (1997) mentioned in Section 4, which establishes the same nonexistence result in two-agent pure exchange economies.

Let $u^{0}\left(x_{1}, \ldots, x_{n}\right)=x_{1} \times \cdots \times x_{n}, u^{1}\left(x_{1}, \ldots, x_{n}\right)=\left(x_{1}\right)^{2} \times x_{2} \times \cdots \times x_{n}$, and $z=$ $f\left(u^{1}, u^{0}, \ldots, u^{0}\right)$. Since $d=f\left(u^{0}, u^{0}, \ldots, u^{0}\right)$, by Lemma 3.3, it is not the case that $z^{1}<$ $d^{1}$ or that $z^{1}>d^{1}$. Since $\lambda^{1} \in(0,1)$ and since $d^{1}=\lambda^{1} \cdot \Omega, z$ is off diagonal, and $z^{1} \in \mathbb{R}_{++}^{m}$. Thus there is $p \in \mathbb{R}_{++}^{m}$ such that $p \cdot\left(z^{1}-d^{1}\right)<0$. Since $z^{1}=\Omega-\sum_{i=2}^{i=n} z^{i}$ and $d^{1}=\Omega-\sum_{i=2}^{i=n} d^{i}$, we have $p \cdot\left(\sum_{i=2}^{i=n} d^{i}-\sum_{i=2}^{i=n} z^{i}\right)<0$. Given $\sigma \in(0,1)$ and $x \in \mathbb{R}_{+}^{m}$, let $\widehat{u}^{1}(x)=\left[\sum_{l \in M} p_{l} \cdot\left(z_{l}^{1}\right)^{1-\sigma} \cdot\left(x_{l}\right)^{\sigma}\right]^{1 / \sigma}$. Note that for any $x \in\left[z^{1}, \Omega\right]$, the vector $q=\left(p_{l} \cdot\left(z_{l}^{1}\right)^{1-\sigma} \cdot\left(x_{l}\right)^{\sigma-1}\right)_{l \in M}$ generates a hyperplane supporting $U C\left(\widehat{u}^{1}, x\right)$ at $x$. As a special case, $p$ generates a hyperplane supporting $U C\left(\widehat{u}^{1}, z^{1}\right)$ at $z^{1}$. Thus since $p \cdot\left(z^{1}-d^{1}\right)<0$, there is $\sigma$ close to 1 such that $\widehat{u}^{1}(d)>\widehat{u}^{1}\left(z^{1}\right)$. Let $\sigma$ be so chosen. Given $\rho \in(-\infty, 1)$, let $\widehat{u}^{0}(\cdot ; \rho)$ be the preference specified in Fact 2 such that $z^{0}=\sum_{i=2}^{i=n} z^{i}$. By Lemma 3.4, $f\left(u^{1}, \widehat{u}^{0}(\cdot ; \rho), \ldots, \widehat{u}^{0}(\cdot ; \rho)\right)=z$ for any $\rho<\rho_{0}$, where $\rho_{0}$ is specified in Fact 2 . Note that as $\rho \longrightarrow-\infty, P^{1}\left(\widehat{u}^{1}, \widehat{u}^{0}(\cdot ; \rho), Z(2, \Omega)\right) \cap U C\left(\widehat{u}^{1}, z^{1}\right)$ converges to $\left[z^{1}, \Omega\right]$. Also note that $f\left(\widehat{u}^{0}(\cdot ; \rho), \widehat{u}^{0}(\cdot ; \rho), \ldots, \widehat{u}^{0}(\cdot ; \rho)\right)=d$ for any $\rho$, so that $\widehat{u}^{1}\left(f\left(\widehat{u}^{1}, \widehat{u}^{0}(\cdot ; \rho), \ldots, \widehat{u}^{0}(\cdot ; \rho)\right)\right) \geq$ $\widehat{u}^{1}(d)>\widehat{u}^{1}(z)$ for any $\rho$. Thus it follows from Pareto-efficiency and strategy-proofness that there is $\rho_{1}$ such that for any $\rho<\rho_{1}, u^{1}\left(f\left(\widehat{u}^{1}, \widehat{u}^{0}(\cdot ; \rho), \ldots, u^{0}(\cdot ; \rho)\right)\right)>u^{1}(z)$. Therefore, for $\rho<\min \left\{\rho_{0}, \rho_{1}\right\}, u^{1}\left(f\left(\widehat{u}^{1}, \widehat{u}^{0}(\cdot ; \rho), \ldots, \widehat{u}^{0}(\cdot ; \rho)\right)\right)>u^{1}\left(f\left(u^{1}, \widehat{u}^{0}(\cdot ; \rho), \ldots, \widehat{u}^{0}(\cdot ; \rho)\right)\right)$. This contradicts strategy-proofness. Q.E.D. 
Proof of Corollary. Suppose that there is an effectively pairwise strategy-proof, Paretoefficient, and nondictatorial rule $f$ on $U \supseteq\left(U^{C} \cap U^{H}\right)^{n}$. Let $\widehat{U}=\left(U^{C} \cap U^{H}\right)^{n}$, and let $\widehat{f}$ be the restriction of $f$ to $\widehat{U}$. Since $f$ is strategy-proof and Pareto-efficient, $\widehat{f}$ is also strategy-proof and Pareto-efficient on $\widehat{U}$. Thus, by Theorem $2, \widehat{f}$ is dictatorial. Without loss of generality, we may assume that agent 1 is a dictator on $\widehat{U}$, that is, for any $u \in \widehat{U}$, $\widehat{f}(u)=f(u)=(\Omega, 0, \ldots, 0)$. We claim that agent 1 is a dictator on the whole $U$, that is, for any $u \in U, f(u)=(\Omega, 0, \ldots, 0)$.

Let $u \in U$, and $\widehat{u} \in \widehat{U}$. Since $f(\widehat{u})=(\Omega, 0, \ldots, 0)$, strategy-proofness for agent $1 \mathrm{im}$ plies that $f^{1}\left(u^{1}, \widehat{u}^{-1}\right)=\Omega$. For otherwise, $u^{1}(f(\widehat{u}))>u^{1}\left(f\left(u^{1}, \widehat{u}^{-1}\right)\right)$. Thus $f\left(u^{1}, \widehat{u}^{-1}\right)=$ $(\Omega, 0, \ldots, 0)$. Then strategy-proofness for agent 2 implies that $f^{2}\left(u^{1}, u^{2}, \widehat{u}^{-\{1,2\}}\right)=0$. For otherwise, $\widehat{u}^{2}\left(f\left(u^{1}, u^{2}, \widehat{u}^{-\{1,2\}}\right)\right)>\widehat{u}^{2}\left(f\left(u^{1}, \widehat{u}^{-1}\right)\right)$. By nonbossiness (Proposition 3 ), we have $f\left(u^{1}, u^{2}, \widehat{u}^{-\{1,2\}}\right)=(\Omega, 0, \ldots, 0)$. Repeating this argument for $j=3, \ldots, n$, we get the result that $f(u)=(\Omega, 0, \ldots, 0)$. This is a contradiction to nondictatorship. Q.E.D.

\section{Subsection 6.4 Proofs of Results in Section 5}

In this subsection, we prove the results in Section 5. First, we introduce a useful notation in allotment economies. Let $Z^{0}=[0, \Omega]$. Given a single-peaked preference $u^{0} \in U^{S}$ and $z^{0} \in Z^{0} \backslash\left\{b\left(u^{0}\right)\right\}$, there is at most one element $\widehat{z}^{0}$ of $Z^{0} \backslash\left\{z^{0}\right\}$ such that $u^{0}\left(\widehat{z}^{0}\right)=u^{0}\left(z^{0}\right)$, and we denote that element by $e\left(u^{0}, z^{0}\right)$ if it exists. The key condition of the proofs of this subsection is also nonbossiness. Lemmas 4.1 and 4.2 below together explain what will happen if a rule is effectively pairwise strategy-proof but not nonbossy. These two lemmas will be employed to establish nonbossiness in Proposition 5 .

Lemma 4.1. Let $f$ be an effectively pairwise pairwise strategy-proof rule. Let $u \in U$, $i \in N$, and $\hat{u}^{i} \in U^{S}$ be such that $u^{i}\left(f^{i}\left(\hat{u}^{i}, u^{-i}\right)\right)=u^{i}\left(f^{i}(u)\right)$ and $\hat{u}^{i}\left(f^{i}\left(\hat{u}^{i}, u^{-i}\right)\right)=\hat{u}^{i}\left(f^{i}(u)\right)$. Denote $D=\left\{j \in N \mid f^{j}\left(\hat{u}^{i}, u^{-i}\right) \neq f^{j}(u)\right\}$ and $D^{\prime}=\left\{j \in N \mid f^{j}(u)=b\left(u^{j}\right)\right\}$. Then (i), (ii), (iii), and (iv) below hold.

(i) $u^{j}\left(f^{j}\left(\hat{u}^{i}, u^{-i}\right)\right)=u^{j}\left(f^{j}(u)\right)$ for any $j \in D$, and $f^{j}\left(\hat{u}^{i}, u^{-i}\right)=f^{j}(u)$ for any $j \in D^{\prime}$.

(ii) If $f^{-i}\left(\hat{u}^{i}, u^{-i}\right) \neq f^{-i}(u)$, then $D \neq \emptyset$, and $\sum_{j \in D} f^{j}\left(\hat{u}^{i}, u^{-i}\right)=\sum_{j \in D} f^{j}(u)$.

(iii) $D \cap D^{\prime}=\emptyset$.

(iv) $e\left(u^{j}, f^{j}(u)\right)$ exists for any $j \in D$, and $\sum_{j \in D} e\left(u^{j}, f^{j}(u)\right)=\sum_{j \in D} f^{j}(u)$.

The condition (i) of Lemma 4.1 follows from Lemma 1.1. The condition (ii) follows directly from the definition of $D$ and resource balance. The condition (iii) follows from the condition (i) and the definition of $D$. The condition (iv) follows from the conditions (i) and (ii).

Lemma 4.2. Let $f$ be an effectively pairwise strategy-proof rule. Let $u \in U, i \in N$, and $\hat{u}^{i} \in U^{S}$ be such that $f^{i}(u)=f^{i}\left(\hat{u}^{i}, u^{-i}\right)$ and $f^{-i}(u) \neq f^{-i}\left(\hat{u}^{i}, u^{-i}\right)$. Then for some preference profile $\tilde{u}$, there are $C \subseteq N$ and $C^{\prime} \subseteq N$ such that

(i) $C \cap C^{\prime}=\emptyset, C \cup C^{\prime}=N, C \neq \emptyset$,

(ii) $C^{\prime}=\left\{j \in N \mid b\left(\tilde{u}^{j}\right)=f^{j}(\tilde{u})\right\}$, and

(iii) $e\left(\tilde{u}^{j}, f^{j}(\tilde{u})\right)$ exists for any $j \in C$, and $\sum_{j \in C} e\left(\tilde{u}^{j}, f^{j}(\tilde{u})\right)=\sum_{j \in C} f^{j}(\tilde{u})$.

Proof of Lemma 4.2. We will construct $C, C^{\prime}$, and $\tilde{u}$ by means of algorithm. Our algorithm consists of Operation, and Cases $0,1,2,3$, and 4 defined below.

Let $D=\left\{j \in N \mid f^{j}\left(\hat{u}^{i}, u^{-i}\right) \neq f^{j}(u)\right\}$ and $D^{\prime}=\left\{j \in N \mid f^{j}(u)=b\left(u^{j}\right)\right\}$. By $f^{i}(u)=$ $f^{i}\left(\hat{u}^{i}, u^{-i}\right)$, we have $u^{i}\left(f^{i}\left(\hat{u}^{i}, u^{-i}\right)\right)=u^{i}\left(f^{i}(u)\right)$ and $\hat{u}^{i}\left(f^{i}\left(\hat{u}^{i}, u^{-i}\right)\right)=\hat{u}^{i}\left(f^{i}(u)\right)$. Therefore, it follows from Lemma 4.1 and $f^{-i}(u) \neq f^{-i}\left(\hat{u}^{i}, u^{-i}\right)$ that $D \cap D^{\prime}=\emptyset, D \neq \emptyset, e\left(u^{j}, f^{j}(u)\right)$ 
exists for any $j \in D$, and $\sum_{k \in D} e\left(u^{k}, f^{k}(u)\right)=\sum_{k \in D} f^{k}(u)$. If $N=D \cup D^{\prime}$, then we set $C$ to be $D, C^{\prime}$ to be $D^{\prime}$, and $\tilde{u}$ to be $u$; and the proof is done. If $N \backslash\left(D \cup D^{\prime}\right) \neq \emptyset$, then we are now in Case 0 below.

Case 0: The following conditions (i'), (ii'), and (iii') hold for $D \subset N$ and $D^{\prime} \subset N$.

(i') $D \cap D^{\prime}=\emptyset, D \cup D^{\prime} \neq N$, and $D \neq \emptyset$.

(ii') $D^{\prime}=\left\{j \in N \mid f^{j}(u)=b\left(u^{j}\right)\right\}$.

(iii') $e\left(u^{j}, f^{j}(u)\right)$ exists for any $j \in D$, and $\sum_{j \in D} e\left(u^{j}, f^{j}(u)\right)=\sum_{j \in D} f^{j}(u)$.

When we are in Case 0, we execute the operation below.

Operation: Pick an agent $j$ from $N \backslash\left(D \cup D^{\prime}\right)$. Let $\hat{u}^{j} \in U^{S}$ be such that $b\left(\hat{u}^{j}\right)=f^{j}(u)$, and let $D^{\prime \prime}=\left\{k \in N \mid f^{k}\left(\hat{u}^{j}, u^{-j}\right) \neq f^{k}(u)\right\}$.

As the result of the operation above, we have $f^{j}\left(\hat{u}^{j}, u^{-j}\right)=f^{j}(u)$ by strategy-proofness, and so that $j \notin D^{\prime \prime}$. Note that one of the four possible cases below must hold.

$$
\begin{aligned}
& \text { Case } 1: \quad f^{-j}\left(\hat{u}^{j}, u^{-j}\right)=f^{-j}(u) \quad \& \quad N=D^{\prime} \cup\{j\} \cup D . \\
& \text { Case } 2: \quad f^{-j}\left(\hat{u}^{j}, u^{-j}\right)=f^{-j}(u) \quad \& \quad N \neq D^{\prime} \cup\{j\} \cup D \text {. } \\
& \text { Case } 3: \quad f^{-j}\left(\hat{u}^{j}, u^{-j}\right) \neq f^{-j}(u) \quad \& \quad N=D^{\prime} \cup\{j\} \cup D^{\prime \prime} \text {. } \\
& \text { Case } 4: \quad f^{-j}\left(\hat{u}^{j}, u^{-j}\right) \neq f^{-j}(u) \quad \& \quad N \neq D^{\prime} \cup\{j\} \cup D^{\prime \prime} \text {. }
\end{aligned}
$$

The discussion and the direction for each of the four cases are given below.

Case 1: It holds that $f\left(\hat{u}^{j}, u^{-j}\right)=f(u)$. Thus, $D^{\prime} \cup\{j\}$ is the set of all agents whose consumptions are equal to their peaks at the profile $\left(\hat{u}^{j}, u^{-j}\right)$, $e\left(u^{k}, f^{k}\left(\hat{u}^{j}, u^{-j}\right)\right)$ exists for any $k \in D$, and $\sum_{k \in D} e\left(u^{k}, f^{k}(u)\right)=\sum_{k \in D} f^{k}(u)$ implies $\sum_{k \in D} e\left(u^{k}, f^{k}\left(\hat{u}^{j}, u^{-j}\right)\right)=\sum_{k \in D} f^{k}\left(\hat{u}^{j}, u^{-j}\right)$. We set $C$ to be $D, C^{\prime}$ to be $D^{\prime} \cup\{j\}$, and the preference profile $\tilde{u}$ to be $\left(\hat{u}^{j}, u^{-j}\right)$. Then, the proof is done.

Case 2: It holds that $f\left(\hat{u}^{j}, u^{-j}\right)=f(u)$. Thus, $D^{\prime} \cup\{j\}$ is the set of all agents whose consumptions are equal to their peaks at the profile $\left(\hat{u}^{j}, u^{-j}\right)$, $e\left(u^{k}, f^{k}\left(\hat{u}^{j}, u^{-j}\right)\right)$ exists for any $k \in D$, and $\sum_{k \in D} e\left(u^{k}, f^{k}(u)\right)=\sum_{k \in D} f^{k}(u)$ implies $\sum_{k \in D} e\left(u^{k}, f^{k}\left(\hat{u}^{j}, u^{-j}\right)\right)=\sum_{k \in D} f^{k}\left(\hat{u}^{j}, u^{-j}\right)$. We reset the notation, and let the new $D^{\prime}$ denote $D^{\prime} \cup\{j\}$, and the new $u$ denote $\left(\hat{u}^{j}, u^{-j}\right)$. Then, we go back to Case 0 above and execute the same operation as above.

Case 3: By $f^{j}\left(\hat{u}^{j}, u^{-j}\right)=f^{j}(u)$, it holds that $u^{j}\left(f^{j}\left(\hat{u}^{j}, u^{-j}\right)\right)=u^{j}\left(f^{j}(u)\right)$ and $\hat{u}^{j}\left(f^{j}\left(\hat{u}^{j}, u^{-j}\right)\right)=\hat{u}^{j}\left(f^{j}(u)\right)$. Thus by Lemma 4.1, $D^{\prime \prime} \cap\left(D^{\prime} \cup\{j\}\right)=$ $\emptyset, D^{\prime \prime} \neq \emptyset, e\left(u^{k}, f^{k}(u)\right)$ exists for any $k \in D^{\prime \prime}$, and $\sum_{k \in D^{\prime \prime}} e\left(u^{k}, f^{k}(u)\right)=$ $\sum_{k \in D^{\prime \prime}} f^{k}(u)$. In addition, $D^{\prime} \cup\{j\}$ is the set of all agents whose consumptions are equal to their peaks at the profile $\left(\hat{u}^{j}, u^{-j}\right)$. We set $C$ to be $D^{\prime \prime}, C^{\prime}$ to be $D^{\prime} \cup\{j\}$, and $\tilde{u}$ to be the preference profile $\left(\hat{u}^{j}, u^{-j}\right)$. Then, the proof is done.

Case 4: By $f^{j}\left(\hat{u}^{j}, u^{-j}\right)=f^{j}(u)$, it holds that $u^{j}\left(f^{j}\left(\hat{u}^{j}, u^{-j}\right)\right)=u^{j}\left(f^{j}(u)\right)$ and $\hat{u}^{j}\left(f^{j}\left(\hat{u}^{j}, u^{-j}\right)\right)=\hat{u}^{j}\left(f^{j}(u)\right)$. Thus by Lemma 4.1, $D^{\prime \prime} \cap\left(D^{\prime} \cup\{j\}\right)=$ $\emptyset, D^{\prime \prime} \neq \emptyset, e\left(u^{k}, f^{k}(u)\right)$ exists for any $k \in D^{\prime \prime}$, and $\sum_{k \in D^{\prime \prime}} e\left(u^{k}, f^{k}(u)\right)=$ $\sum_{k \in D^{\prime \prime}} f^{k}(u)$. In addition, $D^{\prime} \cup\{j\}$ is the set of all agents whose consumptions 
are equal to their peaks at the profile $\left(\hat{u}^{j}, u^{-j}\right)$. We reset the notation, and let the new $D$ denote $D^{\prime \prime}$, the new $D^{\prime}$ denote $D^{\prime} \cup\{j\}$, and the new $u$ denote $\left(\hat{u}^{j}, u^{-j}\right)$. Then, we go back to Case 0 above and execute the same operation as above.

Note that after each operation, a new agent enters $D^{\prime}$. Thus after some finite repetitions of the operation, it must be the case that $N=D \cup\{j\} \cup D^{\prime}$ or $N=D^{\prime} \cup\{j\} \cup D^{\prime \prime}$, that is, Case 1 or Case 3 must hold. Hence, the constructions of $C, C^{\prime}$, and $\tilde{u}$ are completed. Q.E.D.

Proof of Proposition 5. Let $u \in U, i \in N$, and $\hat{u}^{i} \in U^{S}$ be such that $f^{i}(u)=f^{i}\left(\hat{u}^{i}, u^{-i}\right)$. We want to show that $f^{-i}(u)=f^{-i}\left(\hat{u}^{i}, u^{-i}\right)$. Suppose $f^{-i}(u) \neq f^{-i}\left(\hat{u}^{i}, u^{-i}\right)$. By Lemma 4.2, we may assume that there are $C \subseteq N$ and $C^{\prime} \subseteq N$ such that $C \cap C^{\prime}=\emptyset, C \cup C^{\prime}=$ $N, C \neq \emptyset, e\left(u^{j}, f^{j}(u)\right)$ exists for any $j \in C, C^{\prime}=\left\{j \in N: b\left(u^{j}\right)=f^{j}(u)\right\}$, and $\sum_{j \in C} e\left(u^{j}, f^{j}(u)\right)=\sum_{j \in C} f^{j}(u)$.

Note that $C \cap C^{\prime}=\emptyset$ implies that for any $j \in C, e\left(u^{j}, f^{j}(u)\right)<b\left(u^{j}\right)<f^{j}(u)$ or $e\left(u^{j}, f^{j}(u)\right)>b\left(u^{j}\right)>f^{j}(u)$. Thus for each $j \in C$, there is $\bar{u}^{j} \in U^{S}$ such that $b\left(\bar{u}^{j}\right)=\left[e\left(u^{j}, f^{j}(u)\right)+f^{j}(u)\right] / 2$, and

$$
\forall x^{j} \in Z^{0},\left[\bar{u}^{j}\left(f^{j}(u)\right) \geq \bar{u}^{j}\left(x^{j}\right) \Longleftrightarrow u^{j}\left(f^{j}(u)\right) \geq u^{j}\left(x^{j}\right)\right] .
$$

Figure 2 below illustrates the preference $\bar{u}^{j}$. Since $\sum_{j \in C} e\left(u^{j}, f^{j}(u)\right)=\sum_{j \in C} f^{j}(u)$, it follows that

$$
\begin{aligned}
\sum_{j \in C} b\left(\bar{u}^{j}\right) & =\sum_{j \in C}\left[e\left(u^{j}, f^{j}(u)\right)+f^{j}(u)\right] / 2 \\
& =\left[\sum_{j \in C} e\left(u^{j}, f^{j}(u)\right)+\sum_{j \in C} f^{j}(u)\right] / 2 \\
& =\sum_{j \in C} f^{j}(u) .
\end{aligned}
$$

Without loss of generality, let $C=\{1, \ldots, c\}$. We establish by induction logic that for any $k \in C=\{1, \ldots, c\}$, it holds that

(1) $f^{j}\left(\bar{u}^{K}, u^{-K}\right)=f^{j}(u)$ for any $j \in C^{\prime}$, and

(2) $f^{j}\left(\bar{u}^{K}, u^{-K}\right)=f^{j}(u)$ or $f^{j}\left(\bar{u}^{K}, u^{-K}\right)=e\left(u^{j}, f^{j}(u)\right)$ for any $j \in C$, where $K=\{1, \ldots, k\}$ and $\bar{u}^{K}=\left(\bar{u}^{1}, \ldots, \bar{u}^{k}\right)$.

Step 1: We show (1) and (2) in the case that $k=1$. Note that by $(*)$, strategy-proofness implies that $f^{1}\left(\bar{u}^{1}, u^{-1}\right)=f^{1}(u)$ or $f^{1}\left(\bar{u}^{1}, u^{-1}\right)=e\left(u^{1}, f^{1}(u)\right)$. Therefore by (*) again, we have $u^{1}\left(f^{1}\left(\bar{u}^{1}, u^{-1}\right)\right)=u^{1}\left(f^{1}(u)\right)$ and $\bar{u}^{1}\left(f^{1}\left(\bar{u}^{1}, u^{-1}\right)\right)=\bar{u}^{1}\left(f^{1}(u)\right)$. Thus it follows from Lemma 4.1 that $f^{j}\left(\bar{u}^{1}, u^{-1}\right)=f^{j}(u)$ or $\left.f^{j}\left(\bar{u}^{1}, u^{-1}\right)=e\left(u^{j}, f^{j}(u)\right)\right)$ for any $j \in C$. Furthermore, since $b\left(u^{j}\right)=f^{j}(u)$ for any $j \in C^{\prime}$, it also follows from Lemma 4.1 that $f^{j}\left(\bar{u}^{1}, u^{-1}\right)=b^{j}(u)$ for any $j \in C^{\prime}$.

Step 2: As induction hypothesis, assume that

$\left(1^{\prime}\right) f^{j}\left(\bar{u}^{K^{\prime}}, u^{-K^{\prime}}\right)=f^{j}(u)$ for any $j \in C^{\prime}$, and

$\left(2^{\prime}\right) f^{j}\left(\bar{u}^{K^{\prime}}, u^{-K^{\prime}}\right)=f^{j}(u)$ or $f^{j}\left(\bar{u}^{K^{\prime}}, u^{-K^{\prime}}\right)=e\left(u^{j}, f^{j}(u)\right)$ for any $j \in C$, where $K^{\prime}=\{1, \ldots, k-1\}$ and $\bar{u}^{K^{\prime}}=\left(\bar{u}^{1}, \ldots, \bar{u}^{k-1}\right)$.

We show (1) and (2). By $(*)$ and $\left(2^{\prime}\right)$, strategy-proofness implies that $f^{k}\left(\bar{u}^{K}, u^{-K}\right)=$ $f^{k}(u)$ or $f^{k}\left(\bar{u}^{K}, u^{-K}\right)=e\left(u^{k}, f^{k}(u)\right)$. Thus it follows from Lemma 4.1 and $\left(2^{\prime}\right)$ that 
$f^{j}\left(\bar{u}^{K}, u^{-K}\right)=f^{j}(u)$ or $f^{j}\left(\bar{u}^{K}, u^{-K}\right)=e\left(u^{j}, f^{j}(u)\right)$ for any $j \in C$. Furthermore, it also follows from Lemma 4.1 and $\left(1^{\prime}\right)$ that $f^{j}\left(\bar{u}^{K}, u^{-K}\right)=b^{j}(u)$ for any $j \in C^{\prime}$.

We have completed the induction argument. When the result (2) above is applied to the case that $k=c$, it implies that $f^{j}\left(\bar{u}^{C}, u^{-C}\right)=f^{j}(u)$ or $f^{j}\left(\bar{u}^{C}, u^{-C}\right)=e\left(u^{j}, f^{j}(u)\right)$ for any $j \in C$. Since $b\left(\bar{u}^{j}\right)=\left[e\left(u^{j}, f^{j}(u)\right)+f^{j}(u)\right] / 2$ for any $j \in C$, it follows that $f^{j}\left(\bar{u}^{C}, u^{-C}\right) \neq b\left(\bar{u}^{j}\right)$ for any $j \in C \neq \emptyset$. However, by $C \cup C^{\prime}=N$, we have $\sum_{j \in C^{\prime}} b\left(u^{j}\right)+$ $\sum_{j \in C} b\left(\bar{u}^{j}\right)=\sum_{j \in N} f^{j}(u)=\Omega$. This contradicts to respect for unanimity. Q.E.D.

\section{FIGURE 2 ENTERS HERE.}

It is a well-know property of allotment economies ${ }^{20}$ that a rule $f$ is Pareto-efficient if and only if for any $u \in U$,

(i) $\sum_{k \in N} b\left(u^{k}\right) \geq \Omega \Rightarrow\left[\forall k \in N, f^{k}(u) \leq b\left(u^{k}\right)\right]$, and

(ii) $\sum_{k \in N} b\left(u^{k}\right) \leq \Omega \Rightarrow\left[\forall k \in N, f^{k}(u) \geq b\left(u^{k}\right)\right]$.

We employ this property in the proofs of Propositions 6 and 7.

Proof of Proposition 6. Let $f$ be an effectively pairwise strategy-proof rule that respects unanimity. Suppose that $f$ is not Pareto-efficient. We derive a contradiction. We may assume that there are $u \in U$ and $i \in N$ such that $\sum_{k \in N} b\left(u^{k}\right) \geq \Omega$ but $f^{i}(u)>b\left(u^{i}\right)$ since we can treat the opposite case symmetrically. Notice that $\sum_{k \in N} b\left(u^{k}\right) \geq \Omega$ and $f^{i}(u)>b\left(u^{i}\right)$ imply that there is $j \in N$ such that $f^{j}(u)<b\left(u^{j}\right)$. Without loss of generality, let $i=1$ and $j=2$. Denote $N^{\prime}=\{3, \ldots, n\}$.

For each $k \in N^{\prime}$, let $\widehat{u}^{k} \in U^{S}$ be such that $b\left(\widehat{u}^{k}\right)=f^{k}(u)$. Then by strategy-proofness, $f^{3}\left(\hat{u}^{3}, u^{-3}\right)=f^{3}(u)$. So by nonbossiness (Proposition 5), $f\left(\hat{u}^{3}, u^{-3}\right)=f(u)$. Repeating this argument for $k=4, \ldots, n$, we get $f\left(u^{1}, u^{2}, \hat{u}^{N^{\prime}}\right)=f(u)$, where $\hat{u}^{N^{\prime}}=\left(\hat{u}^{3}, \ldots, \hat{u}^{N}\right)$.

Note that $f^{1}(u)-b\left(u^{1}\right) \geq b\left(u^{2}\right)-f^{2}(u)$ or $f^{1}(u)-b\left(u^{1}\right) \leq b\left(u^{2}\right)-f^{2}(u)$. Consider the case that $f^{1}(u)-b\left(u^{1}\right) \geq b\left(u^{2}\right)-f^{2}(u)$. Let $\widehat{u}^{1} \in U^{S}$ be such that $b\left(\widehat{u}^{1}\right)=f^{1}(u)-$ $\left[b\left(u^{2}\right)-f^{2}(u)\right]$ and $\widehat{u}^{1}\left(f^{1}(u)\right)>\widehat{u}^{1}\left(b\left(u^{1}\right)\right)$. Then by strategy-proofness, $f^{1}\left(\widehat{u}^{1}, u^{2}, \hat{u}^{N^{\prime}}\right)=$ $f^{1}\left(u^{1}, u^{2}, \hat{u}^{N^{\prime}}\right)>b\left(\widehat{u}^{1}\right)$. So by nonbossiness (Proposition 5), $f\left(\widehat{u}^{1}, u^{2}, \hat{u}^{N^{\prime}}\right)=f\left(u^{1}, u^{2}, \hat{u}^{N^{\prime}}\right)=$ $f(u)$. However,

$$
b\left(\widehat{u}^{1}\right)+b\left(u^{2}\right)+\sum_{k \in N^{\prime}} b\left(\widehat{u}^{k}\right)=f^{1}(u)+f^{1}(u)+\sum_{k \in N^{\prime}} f^{k}(u)=\Omega .
$$

This contradicts to respect for unanimity. We can similarly derive a contradiction to respect for unanimity in the case that $f^{1}(u)-b\left(u^{1}\right) \leq b\left(u^{2}\right)-f^{2}(u)$ by using the preference $\widehat{u}^{2} \in U^{S}$ be such that $b\left(\widehat{u}^{2}\right)=f^{2}(u)+\left[f^{1}(u)-b\left(u^{1}\right)\right]$ and $\widehat{u}^{2}\left(f^{2}(u)\right)>\widehat{u}^{2}\left(b\left(u^{1}\right)\right)$. Q.E.D.

Proof of Proposition 7. Let $f$ be an effectively pairwise strategy-proof rule that respects unanimity. Suppose that $f$ is not group strategy-proof. Then there are $u \in U, N^{\prime} \subseteq N$, $N_{1} \subseteq N^{\prime}, N_{2} \subseteq N^{\prime}, N_{3} \subseteq N^{\prime}$, and $\widehat{u}^{N^{\prime}} \in U^{N^{\prime}}$ such that

(1) $N_{1} \neq \emptyset$ and $N_{1} \cup N_{2} \cup N_{3}=N^{\prime}$,

(2) $u^{i}\left(f\left(\widehat{u}^{N^{\prime}}, u^{-N^{\prime}}\right)\right)>u^{i}(f(u))$ for any $i \in N_{1}$,

(3) $f^{i}\left(\widehat{u}^{N^{\prime}}, u^{-N^{\prime}}\right)=f^{i}(u)$ for any $i \in N_{2}$, and

(4) $f^{i}\left(\widehat{u}^{N^{\prime}}, u^{-N^{\prime}}\right) \neq f^{i}(u), e\left(u^{i}, f^{i}(u)\right)$ exists and $f^{i}\left(\widehat{u}^{N^{\prime}}, u^{-N^{\prime}}\right)=e\left(u^{i}, f^{i}(u)\right)$ for any $i \in N_{3}$.

\footnotetext{
${ }^{20}$ See Sprumont (1991).
} 
Note that $N_{1}, N_{2}$, and $N_{3}$ are mutually disjoint. Also note that (1) and (2) imply $f\left(\widehat{u}^{N^{\prime}}, u^{-N^{\prime}}\right) \neq f(u)$.

For each $i \in N_{1}$, by (2), there is $\widetilde{u}^{i} \in M\left(u^{i}, f^{i}(u)\right) \cap M\left(\widehat{u}^{i}, f^{i}\left(\widehat{u}^{N^{\prime}}, u^{-N^{\prime}}\right)\right)$. For each $i \in N_{2}$, let $\widetilde{u}^{i} \in U^{S}$ be such that $b\left(\widetilde{u}^{i}\right)=f^{i}\left(\widehat{u}^{N^{\prime}}, u^{-N^{\prime}}\right)=f^{i}(u)$. Then, by applying Fact 1 and nonbossiness (Proposition 5) successively for agents in $N_{1}$ and $N_{2}$, we have (5) $f\left(\widetilde{u}^{N_{1} \cup N_{2}}, u^{-\left(N_{1} \cup N_{2}\right)}\right)=f(u)$. Similarly, we have (6) $f\left(\widetilde{u}^{N_{1} \cup N_{2}}, \widehat{u}^{N_{3}}, u^{-N^{\prime}}\right)=f\left(\widehat{u}^{N^{\prime}}, u^{-N^{\prime}}\right)$.

Without loss of generality, we may assume that

$$
\sum_{k \in N_{1} \cup N_{2}} b\left(\widetilde{u}^{k}\right)+\sum_{k \in N \backslash\left(N_{1} \cup N_{2}\right)} b\left(u^{k}\right) \geq \Omega
$$

since we can apply the symmetric argument to the case of the opposite inequality. Then, Pareto-efficiency (Proposition 6), (5), and (7) together imply that $f^{i}(u) \leq b\left(u^{i}\right)$ for any $i \in N$. Thus, (4) and (6) imply that $f^{i}(u)<b\left(u^{i}\right)<e\left(u^{i}, f^{i}(u)\right)=f^{i}\left(\widehat{u}^{N^{\prime}}, u^{-N^{\prime}}\right)$ for any $i \in N_{3}$. For each $i \in N_{3}$, let $\widetilde{u}^{i} \in U^{S}$ be such that $b\left(\widetilde{u}^{i}\right)=f^{i}\left(\widehat{u}^{N^{\prime}}, u^{-N^{\prime}}\right)=e\left(u^{n^{\prime}}, f^{n^{\prime}}(u)\right)$. Owing to (6), by applying Fact 1 and nonbossiness (Proposition 5) successively for agents in $N_{3}$, we have $f\left(\widetilde{u}^{N^{\prime}}, u^{-N^{\prime}}\right)=f\left(\widehat{u}^{N^{\prime}}, u^{-N^{\prime}}\right)$.

In the rest of the proof, we establish that $f\left(\widetilde{u}^{N^{\prime}}, u^{-N^{\prime}}\right)=f\left(u^{-N^{\prime}}\right)$, which together with $f\left(\widetilde{u}^{N^{\prime}}, u^{-N^{\prime}}\right)=f\left(\widehat{u}^{N^{\prime}}, u^{-N^{\prime}}\right)$ contradicts to $f\left(\widehat{u}^{N^{\prime}}, u^{-N^{\prime}}\right) \neq f(u)$. Without loss of generality, let $N_{3}=\left\{n^{\prime}, \ldots, n^{\prime \prime}\right\}, n^{\prime} \leq n^{\prime \prime}$.

It follows from $(7)$ and $b\left(u^{n^{\prime}}\right)<f^{n^{\prime}}\left(\widehat{u}^{N^{\prime}}, u^{-N^{\prime}}\right)=b\left(\widetilde{u}^{n^{\prime}}\right)$ that

$$
\sum_{k \in N_{1} \cup N_{2}} b\left(\widetilde{u}^{k}\right)+b\left(\widetilde{u}^{n^{\prime}}\right)+\sum_{k \in N \backslash\left(N_{1} \cup N_{2} \cup\left\{n^{\prime}\right\}\right)} b\left(u^{k}\right) \geq \Omega .
$$

Thus, Pareto-efficiency, (8) implies

$$
f^{n^{\prime}}\left(\widetilde{u}^{N_{1} \cup N_{2}}, \widetilde{u}^{n^{\prime}}, u^{-\left(N_{1} \cup N_{2} \cup\left\{n^{\prime}\right\}\right)}\right) \leq b\left(\widetilde{u}^{n^{\prime}}\right)=e\left(u^{n^{\prime}}, f^{n^{\prime}}(u)\right) .
$$

By (4) and (5), strategy-proofness for agent $n^{\prime}$ with the preference $u^{n^{\prime}}$ implies

$$
\begin{aligned}
f^{n^{\prime}}\left(\widetilde{u}^{N_{1} \cup N_{2}}, \widetilde{u}^{n^{\prime}}, u^{-\left(N_{1} \cup N_{2} \cup\left\{n^{\prime}\right\}\right)}\right) & \leq f^{n^{\prime}}\left(\widetilde{u}^{N_{1} \cup N_{2}}, u^{-\left(N_{1} \cup N_{2}\right)}\right)=f^{n^{\prime}}(u), \\
\text { or } f^{n^{\prime}}\left(\widetilde{u}^{N_{1} \cup N_{2}}, \widetilde{u}^{n^{\prime}}, u^{-\left(N_{1} \cup N_{2} \cup\left\{n^{\prime}\right\}\right)}\right) & \geq e\left(u^{n^{\prime}}, f^{n^{\prime}}(u)\right) .
\end{aligned}
$$

By (4) and (5), strategy-proofness for agent $n^{\prime}$ with the preference $\widetilde{u}^{n^{\prime}}$ implies

$$
f^{n^{\prime}}\left(\widetilde{u}^{N_{1} \cup N_{2}}, u^{-\left(N_{1} \cup N_{2}\right)}\right)=f^{n^{\prime}}(u) \leq f^{n^{\prime}}\left(\widetilde{u}^{N_{1} \cup N_{2}}, \widetilde{u}^{n^{\prime}}, u^{-\left(N_{1} \cup N_{2} \cup\left\{n^{\prime}\right\}\right)}\right) \leq e\left(\widetilde{u}^{n^{\prime}}, f^{n^{\prime}}(u)\right) .
$$

Therefore, it holds that

$$
\begin{aligned}
f^{n^{\prime}}\left(\widetilde{u}^{N_{1} \cup N_{2}}, \widetilde{u}^{n^{\prime}}, u^{-\left(N_{1} \cup N_{2} \cup\left\{n^{\prime}\right\}\right)}\right) & =f^{n^{\prime}}\left(\widetilde{u}^{N_{1} \cup N_{2}}, u^{-\left(N_{1} \cup N_{2}\right)}\right)=f^{n^{\prime}}(u), \\
\text { or } f^{n^{\prime}}\left(\widetilde{u}^{N_{1} \cup N_{2}}, \widetilde{u}^{n^{\prime}}, u^{-\left(N_{1} \cup N_{2} \cup\left\{n^{\prime}\right\}\right)}\right) & =e\left(u^{n^{\prime}}, f^{n^{\prime}}(u)\right)=f^{n^{\prime}}\left(\widehat{u}^{N^{\prime}}, u^{-N^{\prime}}\right) .
\end{aligned}
$$

Suppose $f^{n^{\prime}}\left(\widetilde{u}^{N_{1} \cup N_{2}}, \widetilde{u}^{n^{\prime}}, u^{-\left(N_{1} \cup N_{2} \cup\left\{n^{\prime}\right\}\right)}\right)=f^{n^{\prime}}\left(\widehat{u}^{N^{\prime}}, u^{-N^{\prime}}\right)$. We derive a contradiction. Let $\bar{u}^{n^{\prime}} \in U^{S}$ be such that $b\left(u^{n^{\prime}}\right)<b\left(\bar{u}^{n^{\prime}}\right)<b\left(\widetilde{u}^{n^{\prime}}\right)$ and $\bar{u}^{n^{\prime}}\left(f^{n^{\prime}}(u)\right)<\bar{u}^{n^{\prime}}\left(f^{n^{\prime}}\left(\widehat{u}^{N^{\prime}}, u^{-N^{\prime}}\right)\right)$. Figure 3 below illustrates $\bar{u}^{n^{\prime}}$. It follows from $(7)$ and $b\left(u^{n^{\prime}}\right)<b\left(\bar{u}^{n^{\prime}}\right)$ that

$$
\sum_{k \in N_{1} \cup N_{2}} b\left(\widetilde{u}^{k}\right)+b\left(\bar{u}^{n^{\prime}}\right)+\sum_{k \in N \backslash\left(N_{1} \cup N_{2} \cup\left\{n^{\prime}\right\}\right)} b\left(u^{k}\right) \geq \Omega .
$$


Thus by Pareto-efficiency,

$$
f^{n^{\prime}}\left(\widetilde{u}^{N_{1} \cup N_{2}}, \bar{u}^{n^{\prime}}, u^{-\left(N_{1} \cup N_{2} \cup\left\{n^{\prime}\right\}\right)}\right) \leq b\left(\bar{u}^{n^{\prime}}\right) .
$$

By (4) and (5), strategy-proofness for agent $n^{\prime}$ with the preference $u^{n^{\prime}}$ implies

$$
\begin{aligned}
f^{n^{\prime}}\left(\widetilde{u}^{N_{1} \cup N_{2}}, \bar{u}^{n^{\prime}}, u^{-\left(N_{1} \cup N_{2} \cup\left\{n^{\prime}\right\}\right)}\right) & \leq f^{n^{\prime}}\left(\widetilde{u}^{N_{1} \cup N_{2}}, u^{-\left(N_{1} \cup N_{2}\right)}\right)=f^{n^{\prime}}(u), \\
\text { or } f^{n^{\prime}}\left(\widetilde{u}^{N_{1} \cup N_{2}}, \bar{u}^{n^{\prime}}, u^{-\left(N_{1} \cup N_{2} \cup\left\{n^{\prime}\right\}\right)}\right) & \geq e\left(u^{n^{\prime}}, f^{n^{\prime}}(u)\right) .
\end{aligned}
$$

By (4) and (5), strategy-proofness for agent $n^{\prime}$ with the preference $\bar{u}^{n^{\prime}}$ implies

$$
f^{n^{\prime}}\left(\widetilde{u}^{N_{1} \cup N_{2}}, u^{-\left(N_{1} \cup N_{2}\right)}\right)=f^{n^{\prime}}(u) \leq f^{n^{\prime}}\left(\widetilde{u}^{N_{1} \cup N_{2}}, \bar{u}^{n^{\prime}}, u^{-\left(N_{1} \cup N_{2} \cup\left\{n^{\prime}\right\}\right)}\right) \leq e\left(\bar{u}^{n^{\prime}}, f^{n^{\prime}}(u)\right) .
$$

Therefore, it holds that $f^{n^{\prime}}\left(\widetilde{u}^{N_{1} \cup N_{2}}, \bar{u}^{n^{\prime}}, u^{-\left(N_{1} \cup N_{2} \cup\left\{n^{\prime}\right\}\right)}\right)=f^{n^{\prime}}(u)$. Thus

$$
\bar{u}^{n^{\prime}}\left(f^{n^{\prime}}\left(\widetilde{u}^{N_{1} \cup N_{2}}, \bar{u}^{n^{\prime}}, u^{-\left(N_{1} \cup N_{2} \cup\left\{n^{\prime}\right\}\right)}\right)\right)<\bar{u}^{n^{\prime}}\left(f^{n^{\prime}}\left(\widetilde{u}^{N_{1} \cup N_{2}}, \widetilde{u}^{n^{\prime}}, u^{-\left(N_{1} \cup N_{2} \cup\left\{n^{\prime}\right\}\right)}\right)\right),
$$

contradicting strategy-proofness. Thus, it must be the case that

$$
f^{n^{\prime}}\left(\widetilde{u}^{N_{1} \cup N_{2}}, \widetilde{u}^{n^{\prime}}, u^{-\left(N_{1} \cup N_{2} \cup\left\{n^{\prime}\right\}\right)}\right)=f^{n^{\prime}}\left(\widetilde{u}^{N_{1} \cup N_{2}}, u^{-\left(N_{1} \cup N_{2}\right)}\right)=f^{n^{\prime}}(u) .
$$

Hence by nonbossiness (Proposition 5), we have

$$
f\left(\widetilde{u}^{N_{1} \cup N_{2}}, \widetilde{u}^{n^{\prime}}, u^{-\left(N_{1} \cup N_{2} \cup\left\{n^{\prime}\right\}\right)}\right)=f\left(\widetilde{u}^{N_{1} \cup N_{2}}, u^{-\left(N_{1} \cup N_{2}\right)}\right)=f(u) .
$$

Repeating the same argument as above for agents, $i=n^{\prime}+1, \ldots, n^{\prime \prime}$, we get $f\left(\widetilde{u}^{N^{\prime}}, u^{-N^{\prime}}\right)=$ $f\left(u^{-N^{\prime}}\right)$. Q.E.D.

\section{FIGURE 3 ENTERS HERE.}

\section{Concluding Remark}

In this article, we applied effective pairwise strategy-proofness to the three types of economies: public goods economies, pure exchange economies, and allotment economies. Then, new characterization results have been obtained. The implication of effective pairwise strategy-proofness is interesting from the view point of strategic behavior. Although pairwise strategy-proofness is seemingly much weaker than group strategy-proofness, this axiom characterizes many rules that are analyzed by using different axioms in the literature. These results indicate a new direction of the research on strategy-proof requirements. The author believes that the application of effective pairwise strategy-proofness will also generate interesting characterization results in other types of economies.

\section{References}

Barberà S, and Jackson M (1995) Strategy-Proof Exchange. Econometrica 63: 51-87. Barberà S, Massó J, and Serizawa S (1998) Strategy-Proof Voting on Compact Ranges.

Game Econ Beh 25: 272-291.

Benassy JP (1982) The Economics of Market Disequilibrium. Academic Press, New York. Ching S (1994) An Alternative Characterization of the Uniform Rule. Soc Choice Welfare 
11: $131-136$.

Ded R and Ohseto S (1999) Strategy-Proof and Individually Rational Social Choice

Functions for Public Good Economies: A Note. Econ Theory 14: 685-689.

Gibbard A (1977) Manipulation of Voting Schemes: A General Result. Econometrica 45: 439-446.

Hurwicz L (1972) On Informationally Decentralized Systems. In: McGuire C B, and

Radner R (eds) Decision and Organization. North Holland, Amsterdam, pp297-336.

Maskin E (1999) Nash Equilibrium and Welfare Optimality. Rev Econ Stud 66: 23-38

Moulin H (1980) On Strategy-Proofness and Single-Peakedness. Publ Choice 35: 437-455. 305-325.

Musgrave (1985) A Brief History of Fiscal Doctrine. In: A.J. Auerbach and M. Feldstein (eds) Handbook of Public Economics Vol. I. North-Holland pp1-59.

Ohseto S (1997) Strategy-Proof Mechanism in Public Good Economies. Math Soc Sciences 33: $157-183$.

Peleg B and Sudhölter P (1999) Single-Peaked and Coalition Proofness. Rev Econ Design 4: 381-387.

Samuelson P (1954) The Pure Theory of Public Expenditures. Review of Economics and Statistics 36: 387-389.

Satterthwaite M (1975) Strategy-Proofness and Arrow's Conditions: Existence and Correspondence Theorems for Voting Procedures and Social Welfare Functions.

J Econ Theory 10: 187-217.

Satterthwaite M and Sonnenschein H (1981) Strategy-Proof Allocation Mechanisms at Differentiable points. Rev Econ Stud 48: 587-598.

Schummer J (1997) Strategy-Proofness versus Efficiency on Restricted Domains of Exchange Economies. Soc Choice Welfare 14: 47-56.

Schummer J (2000) Manipulation through Bribes. J Econ Theory 91: 180-198.

Serizawa S (1996) Strategy-Proof and Individually Rational Social Choice Functions

for Public Good Economies. Econ Theory 7: 501-512.

Serizawa S (1999) Strategy-Proof and Symmetric Social Choice Functions for Public Good Economies. Econometrica 76: 121-145.

Serizawa S (2002) Inefficiency of Strategy-Proof Rules for Pure Exchange Economies.

J Econ Theory 106: 219-241

Serizawa S and Weymark J (2003) Efficient Strategy-Proof Exchange and Minimum Consumption Guarantees. J Econ Theory 109: 246-263

Sprumont Y (1991) The Division Problem with Single-Peaked Preferences: A

Characterization of the Uniform Rule. Econometrica 59: 509-519.

Zhou L (1991) Inefficiency of Strategy-Proof Allocation Mechanisms in Pure Exchange

Economies. Soc Choice Welfare 8: 247-257. 


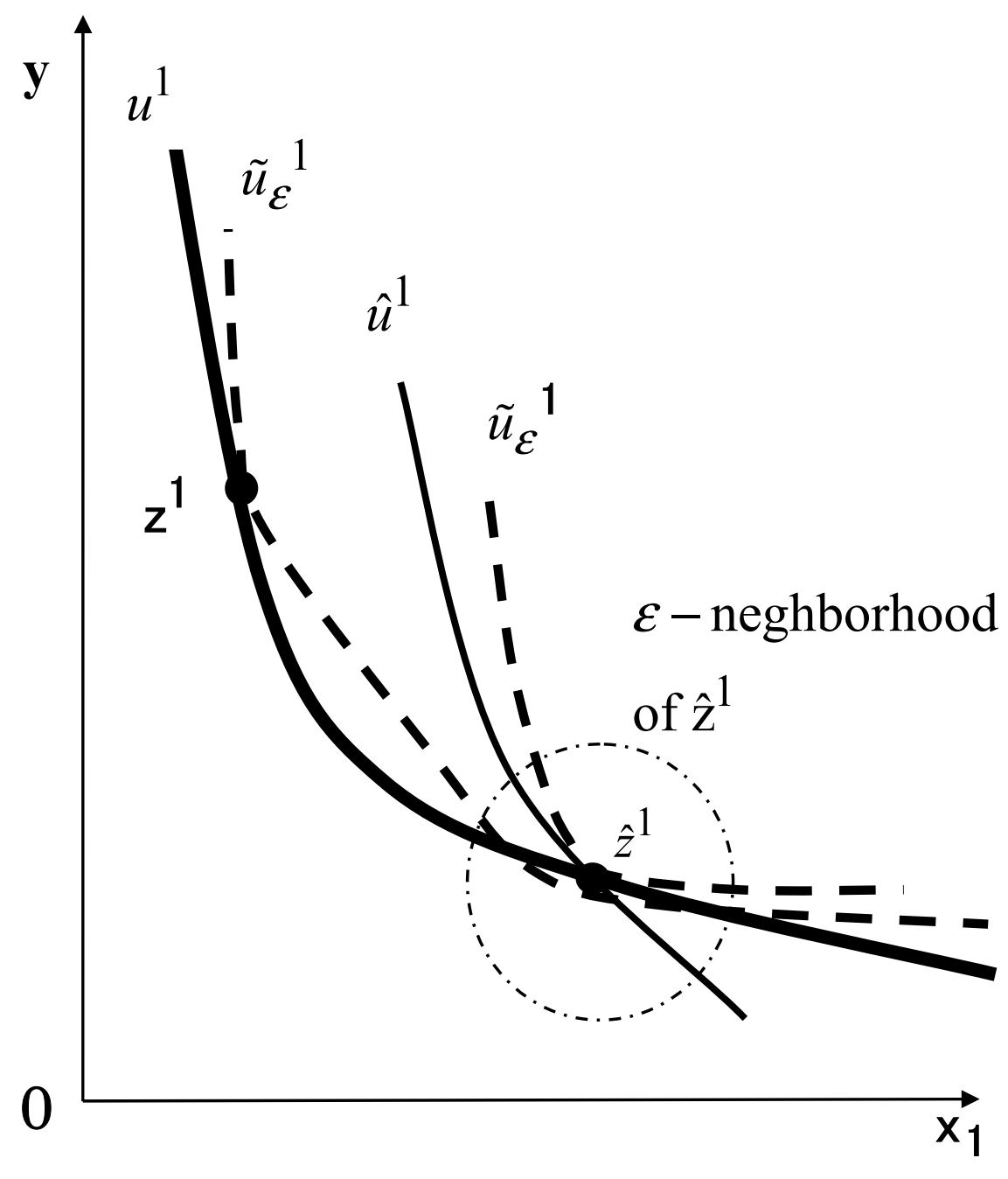

Fig. 1. Illustration of $\tilde{u}_{\varepsilon}{ }^{1}$ in

Proof of Theorem 1 


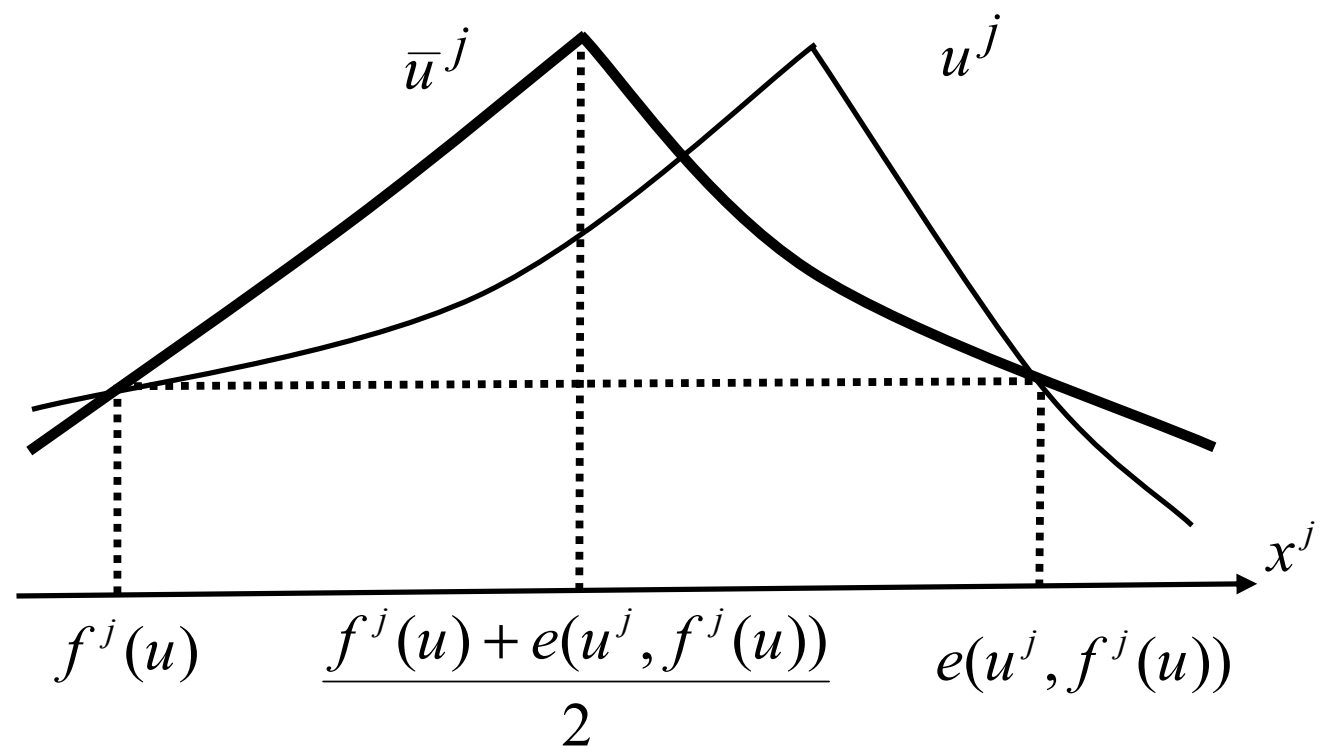

Fig. 2. Illustration of $\bar{u}^{j}$ in Proof of Proposition 5 


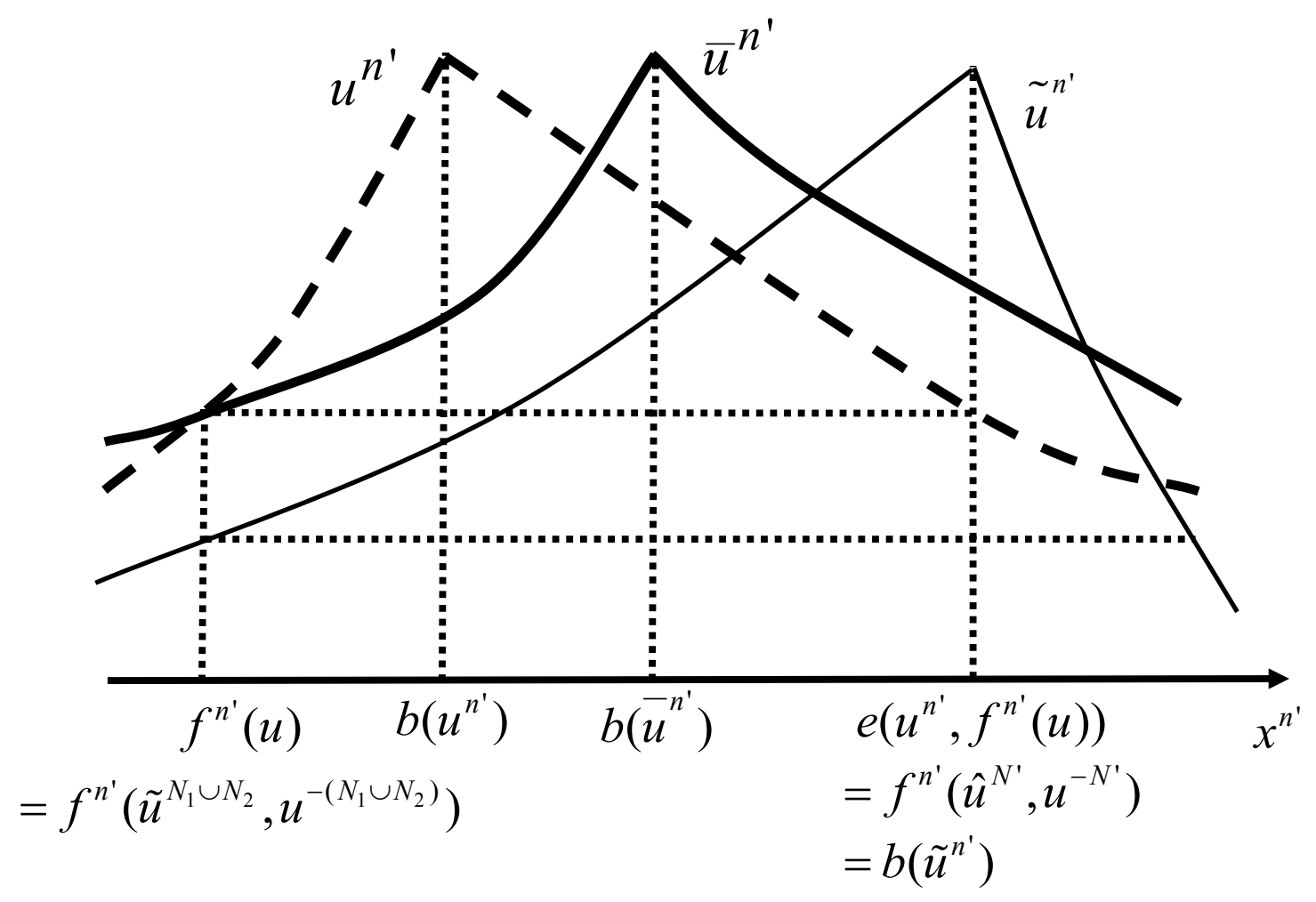

Fig. 3. Illustration of $\bar{u}^{n^{\prime}}$ in Proof of Proposition 7 